\title{
Understanding the Phase Behavior of Coarse-Grained Model Lipid Bilayers through Computational Calorimetry
}

\author{
Jocelyn M. Rodgers, ${ }^{*,+}$ Jesper Sørensen, ${ }^{\neq, \S, \uparrow}$ Frédérick J.-M. de Meyer, ${ }^{\|, \perp}$ Birgit Schiøtt, ${ }^{\neq, \S, \uparrow}$ and \\ Berend Smit ${ }^{*, \|, \perp, \#}$ \\ ${ }^{\dagger}$ Physical Biosciences Division, Lawrence Berkeley National Laboratory, Berkeley, California 94720, United States \\ ${ }^{\ddagger}$ Interdisciplinary Nanoscience Center (iNANO), Aarhus University, Ny Munkegade 118, 8000 Aarhus C, Denmark \\ ${ }^{\S}$ Center for Insoluble Protein Structures (inSPIN), Aarhus University, Langelandsgade 140, 8000 Aarhus C, Denmark \\ "Department of Chemistry, Aarhus University, Langelandsgade 140, 8000 Aarhus C, Denmark \\ "Department of Chemical Engineering, University of California, Berkeley, 101B Gilman Hall, Berkeley, California 94720-1462, \\ United States \\ ${ }^{\perp}$ Materials Science Division, Lawrence Berkeley National Laboratory, Berkeley, California 94720, United States \\ \#Department of Chemistry, University of California, Berkeley, 101B Gilman Hall, Berkeley, California 94720-1462, United States \\ Supporting Information
}

ABSTRACT: We study the phase behavior of saturated lipids as a function of temperature and tail length for two coarse-grained models: the soft-repulsive model typically employed with dissipative particle dynamics (DPD) and the MARTINI model. We characterize the simulated transitions through changes in structural properties, and we introduce a computational method to monitor changes in enthalpy, as is done experimentally with differential scanning calorimetry. The lipid system experimentally presents four different bilayer phases - subgel, gel, ripple, and fluid - and the DPD model describes all of these phases structurally while MARTINI describes a single order-disorder transition between the gel and the fluid phases. Given both models' varying degrees of success in displaying accurate structural and thermodynamic signatures, there is an overall satisfying extent of agreement for the coarse-grained models. We also study the lipid dynamics displayed by these models for the various phases, discussing this dynamics with relation to fidelity to experiment and computational efficiency.

\section{INTRODUCTION}

The past decade has seen an explosion in the development of coarse-grained models for molecular simulation, especially in the context of biophysical study. ${ }^{1}$ One goal of these models is to explain collective phenomena or dynamics that are at too large a scale to be observed in all-atom simulations. Typically, such descriptions come at the cost of a degree of chemical specificity; however, this loss in detail instead allows for more generic features of the systems of interest to be studied. The lipid bilayer is one molecular system that coarse-grained simulations have focused on a great deal, with the intent of drawing biophysical analogies to the cell membrane. ${ }^{2-4}$

A whole host of models have been developed in order to better understand membrane phenomena at varying degrees of detail. $^{2-8}$ We are interested in understanding how well these coarse-grained models can describe the phase behavior of lipid bilayers. Toward this goal, we focus on a subset of these models with two basic features: (i) molecular-level resolution of the bilayer molecules including individual representation of each lipid tail, and (ii) inclusion of explicit solvent with the resolution of several water molecules per water bead. Through this focus we seek to understand both generic conclusions and the trade-offs inherent in different schemes for coarse-graining at a given resolution. With the regular development of new coarse-grained molecular models, a comparative study of this form is merited. The two models we focus on are (i) the soft-repulsive-core model originally developed by Warren and co-workers for dissipative particle dynamics simulations and extended in our research group (DPD), ${ }^{4,9}$ and (ii) the MARTINI force field developed by Marrink and co-workers. ${ }^{7,10}$

The development approaches for coarse-grained models can be different, as can many of the potential energy functional forms. In the section Methods: Molecular Lipid Models, we shall provide a brief summary of the coarse-graining philosophy applied in each case. In comparing the behavior of these models, we focus on the phase behavior of saturated lipid bilayers as a function of temperature and tail length. While the consequences of varying one parameter for this phase behavior has been
Received: August 15, 2011
Revised: December 10, 2011
Published: January 25, 2012 
previously examined with the DPD model, ${ }^{11}$ neither of the models was fully parametrized in order to match these phase phenomena. As such, we place these models on relatively even footing in this comparison, though it has already been established that DPD captures the range of structures characterizing the different bilayer phases. ${ }^{11}$ By studying the phase behavior in as simple a system as possible - a single component lipid bilayer with the length of the lipid varied systematically - we seek to explore the predictive power of these models in detail in a system where substantial experimental data exist.

The tendency when employing coarse-grained systems is to push the limits of the chosen model to study new and biophysically relevant phenomena. As one example, simulations using the MARTINI model have observed the coexistence of the liquid-ordered and liquid-disordered phases in a ternary mixture of saturated lipid, sphingomyelin, and cholesterol, ${ }^{12}$ and have studied the sorting of proteins in phase-separated bilayers. ${ }^{13}$ As another example, simulations using the DPD model have observed many of the "phases" of a saturated lipid-cholesterol bilayer as observed experimentally via structural characterizations ${ }^{14,15}$ and have studied the effect of these membranes on membrane embedded protein interactions. ${ }^{16,17}$ Such studies are crucial and can yield new understanding of complex phenomena.

An implicit assumption behind nearly all lipid bilayer simulation studies seeking understanding of new phenomena is that the model captures the relevant driving forces. While the formation of a gel phase or ripple phase in a single component bilayer may not seem immediately relevant to the membrane-mediated effects on protein-protein interactions or to the coexistence of phases in ternary mixture bilayers, the underlying driving forces behind the reorganization of lipids as temperature changes or as their tails are lengthened are certainly relevant. In addition such phases may be induced by adding a second bilayer component such as cholesterol ${ }^{14,15}$ or an alcohol. ${ }^{18,19}$ By examining the phases formed by the various diacyl phosphatidylcholine bilayers, we are able to not only compare these coarse-grained models to each other but also compare them to a vast body of experimental literature on these phases, as summarized in an excellent review. ${ }^{20}$ The availability of experimental data as a function of tail length allows us to compare the trends present in each coarsegrained model rather than attempting a single comparison to one lipid tail length. Such an analysis of trends is useful since the model parameters are not reoptimized for each distinct lipid and therefore there is intrinsic ambiguity in the exact lipid chain length represented by a coarse-grained lipid of a specified length. Therefore, a detailed study of trends as a function of lipid tail length and temperature could lend further validation of the two CG models and also lend insight into the relevant driving forces of these models.

In this study, beyond comparing the MARTINI and DPD models, we also present the first attempt to characterize the thermodynamics of these phase transitions in solvated, molecular models. Previous studies ${ }^{11,15,21}$ have characterized these phase transitions using a combination of visual inspection and the inflection point for structural properties as a function of temperature. We seek to characterize these transitions by a thermodynamically well-defined quantity: the enthalpy change associated with these phase transformations. Such a quantificiation also allows for direct comparison with experimental measurements arising from differential scanning calorimetry (DSC). As we shall discuss in the section Methods: Calculating DSC Signatures, such approaches have been employed in less molecularly detailed models, but not for models with our considered level of coarse-graining with greater structural fidelity to the true system. We develop a simple computational DSC approach and explore this degree of characterization for our chosen coarse-grained models.

In the section Characterizing Phases of Saturated Lipid Bilayers, we briefly summarize the various bilayer phases and transitions known for the saturated lipids over a range of temperatures, as well as the determination of phase transition temperatures through DSC. Subsequently, we describe our computational approach to DSC. We then summarize the two coarse-grained models that we employ as well as the associated simulation techniques utilized. Under Results, we summarize our findings for each model separately for our quasistatic cooling and heating curves, considering structural parameters, thermodynamic parameters, and lipid dynamics. We then compare these findings more carefully in the Discussion, with an eye toward highlighting the trade-offs inherent to each model.

\section{- CHARACTERIZING PHASES OF SATURATED LIPID BILAYERS}

Known Bilayer Phases. The different phases of pure diacyl phosphatidylcholine bilayers have been studied by a variety of experimental techniques. It is known that lipid bilayers can exist in different phases - the liquid disordered or fluid phase $\left(\mathrm{L}_{\alpha}\right)$, the ripple phase $\left(\mathrm{P}_{\beta^{\prime}}\right)$, the tilted gel phase $\left(\mathrm{L}_{\beta^{\prime}}\right)$, the gel phase $\left(\mathrm{L}_{\beta}\right)$, and the subgel phase $\left(\mathrm{L}_{\mathrm{c}}\right)$ - as shown schematically and from simulation results in Figure $1 .{ }^{22}$ Besides these, a nonlamellar cubic phase and a nonlamellar inverse hexagonal phase have been observed experimentally, ${ }^{23,24}$ and an intermediate bilayer phase $\left(L_{x}\right)$ has been observed for the DLPC bilayers, existing between the ripple and the fluid phase in a narrow temperature range. ${ }^{25,26}$

The transition between the $\mathrm{P}_{\beta^{\prime}}$ and the $\mathrm{L}_{\alpha}$ phase is called the main transition, while the transition between $\mathrm{L}_{\beta^{\prime}}$ and the $\mathrm{P}_{\beta^{\prime}}$ phase is referred to as the pretransition. Additionally, there is a phase transition between the $\mathrm{L}_{\mathrm{c}}$ and $\mathrm{L}_{\beta^{\prime}}$ phases, called the subtransition. ${ }^{27,28}$ The occurrences of these structural transitions are easily quantifiable as there is a shift in at least one measurable parameter, e.g., the volume, ${ }^{28,29}$ the area per lipid, ${ }^{30}$ or the lipid tail order parameter. ${ }^{31,32}$

For the various computational models, we compare structural quantities of the bilayer, similar to examinations previously published for the DPD model of saturated lipids and cholesterol. $^{14,15}$ The liquid-gel transition has also been examined using the MARTINI model by considering the local coordination of beads within the lipid tails, monitoring the extent of lateral 6-fold coordination of tail beads as a gauge of the existence of the gel phase during instantaneous quenches of the bilayer across transition boundaries. ${ }^{21}$ Here we instead consider quasistatic cooling and heating of the MARTINI bilayers, examining more aggregate structural quantities such as area and order parameters, similar to the previous DPD studies.

Enthalpic Signatures of Transition. We also seek to move beyond these structural characterizations to probe a thermodynamic quantity accessible experimentally: the heat capacity. The heat capacity at constant pressure is defined as

$$
C_{p}(T)=\left(\frac{\mathrm{d} Q}{\mathrm{~d} T}\right)_{P}=\left(\frac{\mathrm{d} H}{\mathrm{~d} T}\right)_{P}
$$




\begin{tabular}{|c|c|c|c|c|}
\hline & Subgel $\left(\mathrm{L}_{c}\right)$ & $\operatorname{Gel}\left(\mathrm{L}_{\beta^{\prime}}\right)$ & Ripple $\left(\mathrm{P}_{\beta^{\prime}}\right)$ & Fluid $\left(\mathrm{L}_{\alpha}\right)$ \\
\hline Experiment & 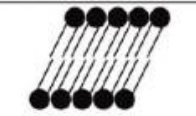 & 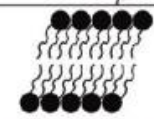 & 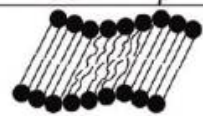 & 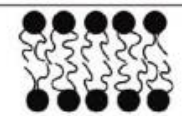 \\
\hline DPD & & & & \\
\hline MARTINI & & & & \\
\hline
\end{tabular}

Figure 1. Diagrams of the various saturated lipid phases. Results from DPD and MARTINI simulations are shown as well as sketched interpretations of the experimental structural consensus. For DPD, the lipid heads are in cyan and the lipid tails are predominantly red, with yellow indicating the final bead in each tail in order to better display the relative tail order in each phase. For MARTINI, the blue beads correspond to choline, red beads represent the phosphate beads, the glycerol groups are shown in pink, and the fatty acid tails are shown in cyan with yellow again indicating the final bead in each tail. As MARTINI does not show all phases, the ripple cell is grayed out and the cells for subgel and gel are a lighter gray as MARTINI displays a single untilted gel phase.

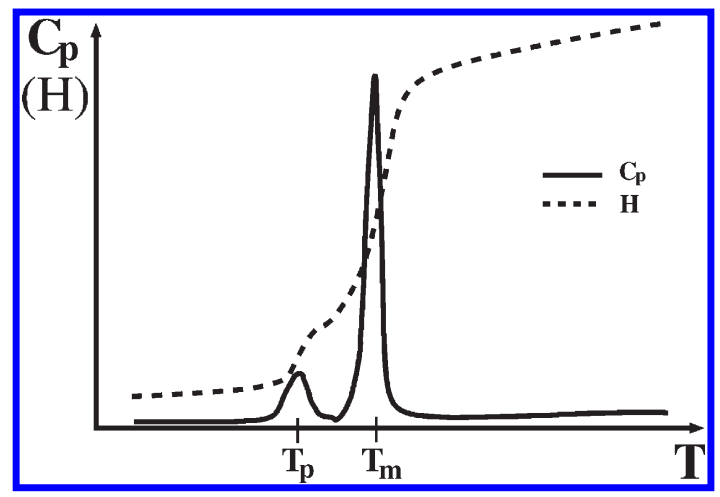

Figure 2. Schematic of the heat capacity $\left(C_{p}\right)$ and enthalpy $(H)$ profiles associated with the pretransition at $T_{\mathrm{p}}$ from the gel phase $\left(\mathrm{L}_{\beta^{\prime}}\right)$ to the ripple phase $\left(\mathrm{P}_{\beta^{\prime}}\right)$ and the main transition at $T_{\mathrm{m}}$ from the ripple phase $\left(\mathrm{P}_{\beta^{\prime}}\right)$ to the liquid phase $\left(\mathrm{L}_{\alpha}\right)$.

During a phase transition, the enthalpy increases sharply, resulting in a much higher heat capacity during the transition. Figure 2 provides a schematic of the expected enthalpy and heat capacity profiles for a phospholipid system undergoing the pretransition at $T_{\mathrm{p}}$ and the main transition at $T_{\mathrm{m}}$. These measured transitions are not infinitely sharp, at least partly due to the finite-sized nature of any phospolipid vesicle. As the names of each transition might suggest, the pretransition corresponds to the weaker, more diffuse peak at lower temperatures and the main transition corresponds to the sharp peak at higher temperatures. A subtransition at $T_{\mathrm{s}}$ would also be visible at lower temperatures. All three of these transitions have experimentally determined signatures of a finite-size first-order phase transition, with an associated latent heat as measured by $\Delta H$. Experimental determination of these heat capacities is not a matter of directly measuring the heat necessary to raise the temperature of a system containing water and vesicles. Rather the DSC technique is required in order to observe the enthalpic signatures of these transitions. ${ }^{33,34}$

Differential Scanning Calorimetry. DSC maintains a cell containing the phospholipid vesicle sample and a reference cell containing only water at the same temperature. The system then tracks the difference in heat needed as the temperatures of two systems are increased simultaneously at a constant rate ranging typically from 0.1 to $1.0 \mathrm{~K} / \mathrm{s}$. This difference in heat required per kelvin increase is essentially the heat capacity of the bilayer system. A tandem measurement of heat uptake is required because otherwise the heat uptake of the bilayer system would be drowned out by the heat uptake of the surrounding aqueous solvent. This experiment-based subtraction of heating effects for the membrane and the solvent allows a focus on the enthalpy changes of the bilayer. ${ }^{22}$

Experimental Data for Comparison. Here we characterize the thermodynamics of the various first-order transitions by $T_{\text {trans }}$ and $\Delta H_{\text {trans }}$ as would be done for macroscopic systems. In the macroscopic limit, these two pieces of information alone are sufficient as the two phases involved in the transition are in equilibrium at $T_{\text {trans, }}$ resulting in $\Delta G_{\text {trans }}=0$ and $\Delta H_{\text {trans }}=$ $T_{\text {trans }} \Delta S_{\text {trans }}$. Koynova and Caffrey compiled both $T_{\text {trans }}$ and $\Delta H_{\text {trans }}$ across many experimental measurements reported in the literature in order to present the trends in the relevant phase changes. ${ }^{20}$ Shown in Figure 3 are the compiled data from that review for transition temperatures as well as the associated changes in enthalpy, with error bars based on variation across the compiled data. Basic trends are evident from this data: (1) both the main and pretransition temperatures increase as a function of acyl chain length while the subtransition temperature increase is less substantial, (2) the pretransition disappears for larger chain lengths, (3) the enthalpy signature for the main transition $\left(\Delta H_{\mathrm{m}}\right)$ is substantially larger than that for the pretransition $\left(\Delta H_{\mathrm{p}}\right)$ and the subtransition $\left(\Delta H_{\mathrm{s}}\right)$ and increases with increasing tail length, and (4) $\Delta H_{\mathrm{s}}$ is smaller than $\Delta H_{\mathrm{m}}$ and essentially constant while $\Delta H_{\mathrm{p}}$ is quite small and nearly zero.

\section{METHODS: CALCULATING DSC SIGNATURES}

Motivated by the concept behind experimental DSC measurements and in hopes of comparing the various models' success in replicating the trends evident in Figure 3, we propose a simple implementation for determining $C_{p}$ based on simulation data. This allows us to obtain a quantitative thermodynamic signature indicative of a first-order phase transition, in essence the "thermodynamic" analogue of examining the variation of structural features with temperature. The heat capacity signature has been studied for more coarse-grained models in greater detail as described briefly below. However, to the best of our knowledge, we are the first to attempt this for lipid bilayer models with explicit solvent. 


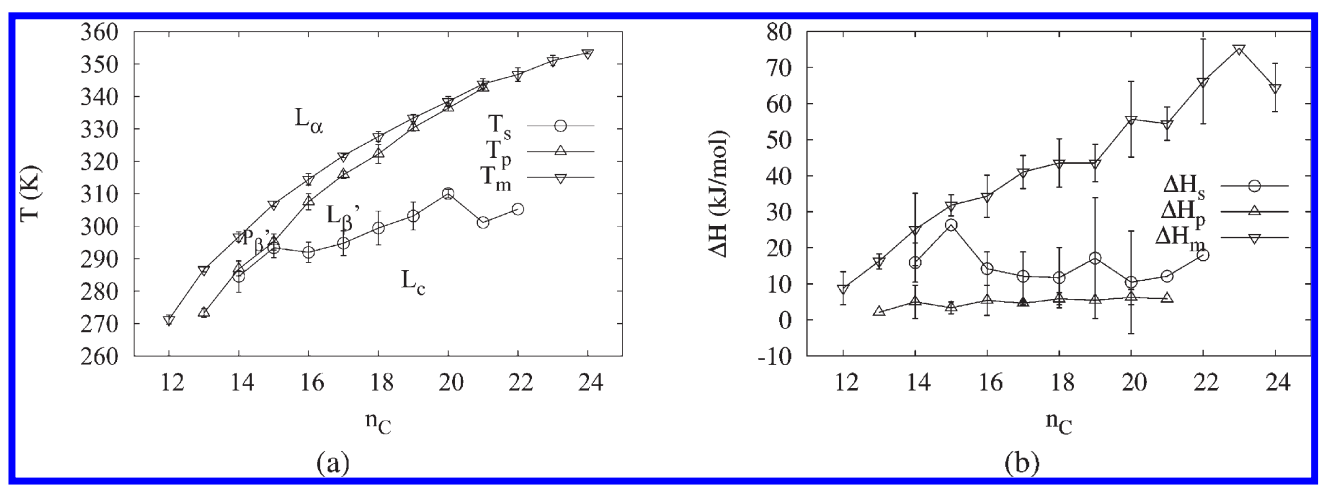

Figure 3. Graphical view of the compilation of experimental data for saturated diacyl phosphatidylcholines reviewed by Koynova and Caffrey. ${ }^{20}$ Data points and error bars are as reported in the article, and lines serve as a guide to the eye. The transition temperatures are shown in (a) and the associated enthalpy changes are displayed in (b), both as a function of the number of carbon atoms in the acyl chain $\left(n_{\mathrm{C}}\right)$.

The determination of phase boundaries based on heat capacity relies on the statistical thermodynamics relation between the heat capacity and the fluctuations in the enthalpy:

$$
C_{p}(T)=\left(\frac{\mathrm{d}\langle H\rangle}{\mathrm{d} T}\right)_{P}=\frac{\left\langle H^{2}\right\rangle-\langle H\rangle^{2}}{R T^{2}}
$$

Many groups as summarized in ref 22 have studied the gel-fluid transitions using this equation and lattice-based Monte Carlo simulations. The simplest, Ising-like models assume that a lipid can be in two states - a gel state and a fluid state - with an interaction parameter indicating the balance between gel-liquid, gel-gel, and liquid-liquid interactions. ${ }^{35}$ This parameter can be optimized to reproduce the experimentally observed heat capacity curve. ${ }^{36}$ There exist a host of refinements to this lattice-based approach, allowing for the description of the pretransition and the main transition as well as many-component bilayers. ${ }^{37,38} \mathrm{In}$ developing these models, the existence of both "gel-like" and "liquid-like" lipids is a specified feature rather than a consequence of lower-level molecular features.

Here, we seek to observe exactly these transitions from molecular-level models that support spontaneous formation of a gel phase and a liquid phase as temperature is varied, rather than positing the existence of these phases a priori. This has been achieved for an intermediate level of molecular detail: a solventfree molecular model for single tail lipids based on density functional theory has recently been studied by Hömberg and Müller and carefully shown to exhibit a first-order phase transition between gel and liquid. ${ }^{39}$ Umbrella sampling over the lipid tail order parameter is required to unambiguously determine the transition along the effective parameter representing tail packing density. Such careful studies based on a molecular density functional yield interesting insights, yet rely on the absence of the solvent and the use of density functional theory in order to make such an exhaustive study computationally reasonable.

A computational consequence of our studying a solventinclusive model is that the fluctuation formula in eq 2 is no longer feasible for straightforward application during simulation. One reason for this is simply that, much as the solvent heat capacity signal would drown out the bilayer heat capacity in experiments, we need an approach to subtract out solvent contributions. The fluctuation formula leads to mixing of terms in the quadratic average and thus does not lead to easily separable contributions. A second reason for this is that a biased sampling technique similar to that used in ref 39 is required to attain sufficient sampling of fluctuations between states in the transition region. Such biased sampling in a fully solvated system is more computationally costly than merited as we are seeking a simple signature with which to survey a range of lipid models. Biased sampling would be a far more accurate way to determine phase boundaries in the statistical-mechanical sense, avoiding possible issues of hysteresis; however, we instead pursue an alternate computational calorimetry which is conceptually straightforward and a quite reasonable approach for a broad assay of coarsegrained models.

As the essence of DSC lies in subtracting the solvent background contribution, we first define

$$
H_{\text {lip }}=H-H_{\text {wat }}^{(0)}=\langle U\rangle+P\langle V\rangle-H_{\text {wat }}^{(0)}
$$

where $H_{\text {wat }}^{(0)}$ is explicitly the reference enthalpy for the same number of water molecules as in this bilayer system if they were instead in a bulk water scenario. With this definition of $H_{\text {wat }}^{(0)}$ we avoid ambiguous choices of which water beads are in the solvation shell of the bilayer yet still include the differential solvation effects of the nearby water molecules on the total lipid-water system enthalpy. Both $\langle U\rangle$ and $\langle V\rangle$ are averages for the entire simulation box; subtraction of $H_{\text {wat }}^{(0)}$ removes the corresponding bulk water contributions to potential energy and to volume. By construction, therefore, $H_{\text {lip }}$ is the enthalpy of the interfacial bilayer system, including the contributions of the solvation shell water relative to bulk water. This enthalpy includes the relative volume differences between solvation shell water and bulk water as well as all interaction energy differences between bulk water and solvation shell water resulting from interactions between lipids and solvation shell waters, among solvation shell waters, and between solvation shell waters and bulk waters. This definition of $H_{\text {lip }}$ in eq 3 computationally encompasses the essence of DSC. In this study, we define $U$ as simply the potential energy since solely the potential energy contributes to enthalpy changes during simulated phase transitions. As a consequence of the purely classical model employed, the kinetic energy contributions to $C_{p}$ will be the ideal quantity $(3 / 2) N_{\text {bead }} k_{\mathrm{B}}$ with no temperature dependence.

Simulation of computational models also allows us to extract the various contributions to $\langle U\rangle$ and $\langle V\rangle$. The contributions to $\langle U\rangle$ may be decomposed as

$$
\begin{aligned}
\langle U\rangle= & \left\langle U_{\mathrm{ww}}\right\rangle+\left\langle U_{\mathrm{wh}}\right\rangle+\left\langle U_{\mathrm{wt}}\right\rangle+\left\langle U_{\mathrm{hh}}\right\rangle+\left\langle U_{\mathrm{ht}}\right\rangle \\
& +\left\langle U_{\mathrm{tt}}\right\rangle+\left\langle U_{\text {bonded }}\right\rangle
\end{aligned}
$$




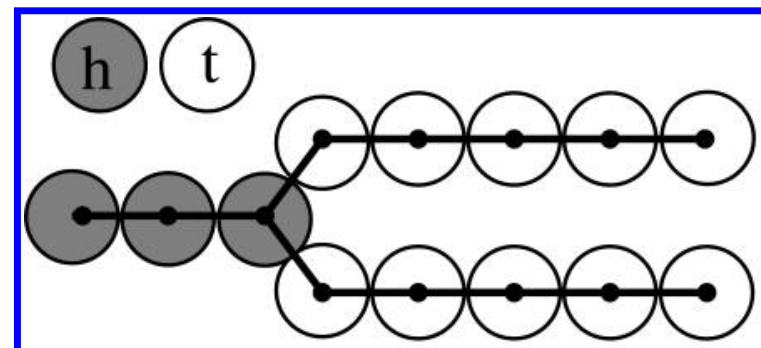

(a) DPD Topology

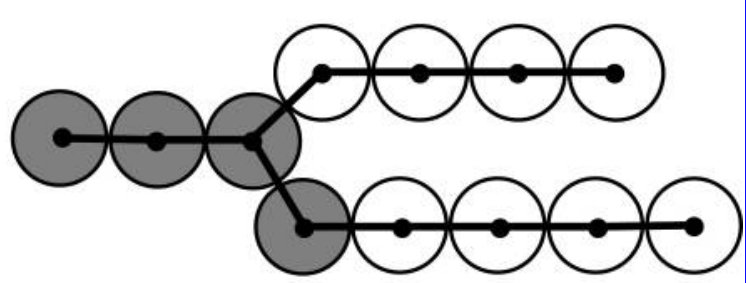

(b) MARTINI Topology

Figure 4. Both molecular coarse-grained models condense the 100-200 atoms in saturated diacyl phosphatidylcholines into three-four headgroup beads and two tails of three-seven tailgroup beads. The DPD model does not distinguish between the individual headgroup beads and tailgroup beads, whereas MARTINI adds a degree of chemical specificity, defining three distinct headgroup beads.

where $U_{i i}$ represent the pair energy contributions due to interactions between heads $(\mathrm{h})$, tails $(\mathrm{t})$, and water $(\mathrm{w})$ beads, and $U_{\text {bonded }}$ represent the intramolecular potential energies for connectivities. Building on this decomposition, we may define "pseudoenthalpies" which have no correspondence to experimentally measurable heat uptakes, but may aid in interpretation of the changes occurring. One such pseudoenthalpy is

$$
\begin{aligned}
H_{\text {tail }}= & \left\langle U_{\mathrm{wt}}\right\rangle+\left\langle U_{\mathrm{ht}}\right\rangle+\left\langle U_{\mathrm{tt}}\right\rangle+\left\langle U_{\text {bonded }}\right\rangle \\
& +P\left\langle V_{\text {tail }}\right\rangle
\end{aligned}
$$

where only energetic contributions involving lipid tail constituents are represented and $V_{\text {tail }}$ is simply the product of the bilayer area with the hydrophobic thickness: $V_{\text {tail }} \equiv\langle A\rangle\left\langle d_{\text {tail }}\right\rangle$. This pseudoenthalpy focuses on the lipid tail since lipid tails are known to undergo dramatic rearrangements during the bilayer transitions and the generalized liquid-gel transition seems dominated by an order-disorder transition in the lipid tails. Of course, only the overall lipid enthalpy has an experimental analogue and thermodynamic meaning. In our analysis of both lipid models, we predominantly examine the DSC-analogue enthalpy $H_{\text {lip }}$, though the tail pseudoenthalpy $H_{\text {tail }}$ proves helpful in interpreting DPD results.

\section{METHODS: MOLECULAR LIPID MODELS}

In this work we use two different coarse-graining approaches: the soft-repulsive-core model (DPD) of Smit and co-workers ${ }^{4,40}$ and the MARTINI model ${ }^{7,10}$ of Marrink and co-workers. In the subsections DPD Model and MARTINI Model we highlight the differences between the two models, both in coarse-graining philosophy and in the choice of functional forms. These two models are interesting to compare as they both use a similar theme for the molecular topology of the lipid, schematically represented for each in Figure 4. This diagram distinguishes head $(\mathrm{h})$ beads from tail $(\mathrm{t})$ beads, which is the sole chemical specificity retained in the DPD model, allowing for representation of the hydrophobicity of the tail beads and the hydrophilicity of the head beads. MARTINI retains further chemical details in the headgroup with separate beads for the phosphate, the choline, and the two glycerol groups, as well as representing the asymmetry in connecting the phosphatidylcholine headgroup with the glycerols. Yet MARTINI still overall has a hydrophilic head and a hydrophobic tail, as the actual lipids do as well.

DPD Model. The DPD model describes the nonbonded interactions between the head $(\mathrm{h})$, tail $(\mathrm{t})$, and water $(\mathrm{w})$ beads using a purely soft repulsive potential with a cutoff: ${ }^{9}$

$$
\mathbf{F}_{i j}^{\mathrm{C}}= \begin{cases}a_{i j}\left(1-r_{i j} / R_{\mathrm{c}}\right) \mathbf{e}_{i j} & \left(r_{i j}<R_{\mathrm{c}}\right) \\ 0 & \left(r_{i j} \geq R_{\mathrm{c}}\right)\end{cases}
$$

This highly simplified potential, which is essentially a continuous analogue of the Flory-Huggins model, ${ }^{9,41}$ truly retains only the relative hydrophobicity and hydrophilicity of the constituent beads with any more detailed interactions such as electrostatics included only in an averaged effective sense. The softness of the potential also allows for much a larger time step than is commonly used in atomistic molecular dynamics (MD) as discussed in the Methods: Simulation Techniques section. In eq $6, a_{i j}$ is a parameter expressing the maximum repulsion strength and varies depending on the type of the two beads interacting, $r_{i j}$ is the distance between the two beads, $R_{c}$ is the cutoff giving the range of the interactions, and $\mathbf{e}_{i j}$ is the unit vector pointing between the two particles.

This model is often described only in the context of the simulation technique - dissipative particle dynamics (DPD) - but the model may be appreciated on its own merits as well. Each bead represents approximately three atomic groupings; one water bead represents three water molecules and one tail bead is roughly three methyl(ene) groups. The length scale $R_{\mathrm{c}}$ is set to $0.646 \mathrm{~nm}$, so a density of 3.0 in reduced DPD units corresponds to typical bulk water density at ambient conditions. The parameters $a_{\mathrm{ww}}, a_{\mathrm{wh}}, a_{\mathrm{wt}}, a_{\mathrm{hh}}, a_{\mathrm{ht}}$, and $a_{\mathrm{tt}}$ may be predominantly derived by matching to Flory-Huggins theory, ${ }^{9,41}$ though the parameter $a_{\mathrm{hh}}$ was set by a more extensive study of the emergent properties of the model. ${ }^{11,42}$ Various harmonic bond and bond angle interactions are set so as to match typical molecular flexibility in all-atom models. ${ }^{43}$

MARTINI Model. The coarse-graining procedure in the MARTINI model is based on a four-to-one mapping of non-hydrogen atoms into beads. ${ }^{10}$ MARTINI 2.0 has four main bead types polar, nonpolar, apolar, and charged - each of which is subdivided into a total of 18 subtypes $^{7}$ to allow for a more accurate representation of the underlying chemical specificity of the atoms collected in the beads.

The interaction parameters between beads is subdivided into bonded and nonbonded interactions as in the DPD model. The bond is described using a weak harmonic potential between the two bonded beads, and an angular potential between consecutive bonded beads describes chain flexibility. ${ }^{10,40}$ The MARTINI model differs fundamentally from the DPD model in its description of nonbonded interactions. Similar to atomistic models, the 
MARTINI model includes van der Waals (vdW) and chargecharge interactions separately. The vdW term is represented by a shifted Lennard-Jones $12-6$ potential: ${ }^{10}$

$$
U_{\mathrm{LJ}}(r)=4 \varepsilon\left[\left(\frac{\sigma}{r}\right)^{12}-\left(\frac{\sigma}{r}\right)^{6}\right]
$$

$\sigma$ is the distance between two particles where the pair energy is 0 and $\sigma \cdot 2^{1 / 6}$ is the distance at which the pair energy is minimal and interactions change from repulsive to attractive. The length scale $\sigma$ is predominantly set to $0.47 \mathrm{~nm}$. The parameter $\varepsilon$ is the strength of the attraction at $r=\sigma \cdot 2^{1 / 6}$ and varies depending on the identity of the two beads interacting. The $\varepsilon$ values are adjusted to roughly match the free energies of hydration, vaporization, and oil-water partitioning for various molecular building blocks. Simultaneously, partially charged groups in the lipid head interact via a Coulomb potential given in SI units as

$$
U_{\mathrm{el}}(r)=\frac{q_{i} q_{j}}{4 \pi \varepsilon_{0} \varepsilon_{\mathrm{r}} r}
$$

where $q_{i}$ and $q_{j}$ are the (partial) charges of the two interacting beads. The relative dielectric screening constant $\varepsilon_{\mathrm{r}}$ in MARTINI is parametrized to 15 , in order to implicitly take into account the screening due to the water dipoles. ${ }^{7}$ We study lipid bilayers solvated with both this original MARTINI water model and a recently updated water model. This recent update now also includes polarizability in the water beads via a Drude-like oscillator. ${ }^{44}$ As such, charges now exist not only in the lipid headgroups but also in the polarizable groups within the solvent beads, and the relative dielectric constant $\varepsilon_{\mathrm{r}}$ has been consequently adjusted closer to a lower value of 2.5 since the simulated solvent polarization accounts for a majority of the dielectric screening. At this point it is important to mention that in the MARTINI implementation of these charges and polarization, either a smoothed spherical cutoff of similar range as the cutoff radius for the LennardJones interaction or full treatment of the long-ranged interaction has been used. The truncation makes the simulations much more efficient, so we employ this here as we are not examining charge density effects themselves in the simulations and the results for many properties with and without long-ranged interactions were found to be similar for this model. ${ }^{44}$ However, this is not strictly correct and may lead to errors; ${ }^{45,46}$ to capture the true longrange character of charges and polarization, it is essential to use special techniques such as Ewald summation or particle mesh methods. $^{47}$

\section{METHODS: SIMULATION TECHNIQUES}

In this section, we describe the different simulation techniques used for each model. The DPD model is simulated via an inhouse hybrid DPD-Monte Carlo simulation code. The MARTINI force field is commonly simulated using the GROMACS MD simulation engine. ${ }^{48}$ Some of the differences in the simulation techniques employed are a result of the typical simulation package used for each model, and some are a direct result of the distinct forms of the model potentials. However, the differences in simulation technique outlined do not alter the equilibrium results obtained as they are all valid approaches for attaining ensemble averages.

DPD Model. The DPD model is typically simulated, as the name suggests, with dissipative particle dynamics, using a modified velocity Verlet integration algorithm. ${ }^{9,49}$ This dynamics simulation technique is a momentum-conserving analogue of Brownian dynamics which applies the random and dissipative forces on a pairwise basis, with the magnitudes of each force chosen in order to maintain the Boltzmann distribution and thereby sample the canonical ensemble. ${ }^{9,49}$

In order to allow variations in bilayer area over the different bilayer phases and also to allow differing degrees of hydration at the bilayer-water interface, we combine dissipative particle dynamics with a Monte Carlo simulation algorithm designed to simulate the constant-normal-pressure, constant-surfacetension ensemble $\left(N P_{\perp} \gamma T\right)$. This hybrid DPD-MC algorithm has been described in detail elsewhere. ${ }^{15,50}$ We simulate at $\gamma=0$ and $P_{\perp}=22.28$ in the reduced units of the DPD model, as explained in ref 50. In these hybrid DPD-MC simulations, $20 \%$ of the Monte Carlo cycles maintain zero surface tensions, $20 \%$ of the MC cycles sample a constant normal pressure, and $60 \%$ of the Monte Carlo cycles are DPD trajectories of varying length. These DPD trajectories within the MC simulations are propagated with a time step $\Delta t=0.03 t_{\text {red }} \approx 51$ fs for an $n_{\text {steps }}$ chosen uniformly between 1 and 50 time steps. For our calculations of the diffusion constant, we simulate using solely dissipative particle dynamics at the average simulation box size determined using the $N P_{\perp} \gamma T$ hybrid DPD-MC approach. This switch in simulation technique is necessitated since, at each $\mathrm{MC}$ cycle in hybrid DPD-MC, the velocities of particles are redrawn from the Maxwell-Boltzmann distribution in order to obey detailed balance.

The time step of $\Delta t=0.03 t_{\text {red }}$ is larger than that typically employed in molecular dynamics simulation of potentials like Lennard-Jones, due to the softness of the DPD potential. We note that in principle the strength of the harmonic bonding interactions may place a tighter upper bound on the allowable time step than suggested by the original analysis of Groot and Warren for the water model. ${ }^{51}$ However, in our studies, we couple harmonic bond forces that are weaker than those explored in ref 51 with an already moderate choice of $\Delta t$, suggesting that the DPD algorithm should thermostat reasonably well.

From hereon, the units of DPD results shall be given in physical units as converted from the reduced units most natural for simulating the DPD model, with the conversions explained further in the Supporting Information. The conversions are not entirely unambiguous; however, reporting our results in physical units is useful for comparing with MARTINI results and with experimental data and these conversions do have a rational basis in the model.

MARTINI Model. The MARTINI model is simulated using molecular dynamics via the GROMACS package, employing the leapfrog Verlet algorithm. ${ }^{10}$ This dynamics is maintained at constant temperature and pressure via coupling to a Berendsen thermostat and a Berendsen barostat at 1 bar. ${ }^{52}$ Application of the barostat is decoupled in the lateral and normal directions, in order to simulate at zero surface tension. Such barostating is often employed to simulate a tensionless system, and its general applicability is further explored elsewhere. ${ }^{53}$ The simulation parameters were kept at default using the example DPPC bilayer from the MARTINI Web site, ${ }^{4}$ although we reduced the time step from 40 to $30 \mathrm{fs}$ and increased the pairlist cutoff radius to $1.4 \mathrm{~nm}$, as suggested in recent evaluations of the model. ${ }^{5,56}$ The electrostatics and vdW forces were treated by shifted Coulomb and Lennard-Jones potentials, respectively. Again, we note that such an approximation for 
Table 1. Simulation Summary

\begin{tabular}{|c|c|c|c|c|c|c|c|c|}
\hline \multicolumn{9}{|c|}{ DPD Simulations } \\
\hline keyword $^{a}$ & \multicolumn{2}{|c|}{$\operatorname{lipid}^{b}$} & W type ${ }^{c}$ & \multirow{2}{*}{$\begin{array}{r}N_{\text {lip }}{ }^{d} \\
400\end{array}$} & \multirow{2}{*}{$\begin{array}{l}N_{\text {wat }}^{d} \\
10000\end{array}$} & \multirow{2}{*}{$\begin{array}{c}T_{\text {start }}{ }^{e}(\mathrm{~K}) \\
340.7\end{array}$} & \multirow{2}{*}{$\begin{array}{c}T_{\text {end }}^{e}(\mathrm{~K}) \\
275.4\end{array}$} & \multirow{2}{*}{$\begin{array}{c}|\Delta T|^{e}(\mathrm{~K}) \\
1.1-5.4\end{array}$} \\
\hline cool_C14_DW & $h_{3}\left(t_{4}\right)_{2}$ & (C14:0) & DPD & & & & & \\
\hline heat_C14_DW & $h_{3}\left(t_{4}\right)_{2}$ & $(\mathrm{C} 14: 0)$ & DPD & 400 & 10000 & 275.4 & 329.8 & $1.1-5.4$ \\
\hline cool_C14_DW & $h_{3}\left(t_{4}\right)_{2}$ & $(\mathrm{C} 14: 0)$ & DPD & 900 & 22500 & 329.8 & 275.4 & $1.1-5.4$ \\
\hline heat_C14_DW & $h_{3}\left(t_{4}\right)_{2}$ & (C14:0) & DPD & 900 & 22500 & 329.8 & 329.8 & $1.1-5.4$ \\
\hline cool_C14_DW & $h_{3}\left(t_{4}\right)_{2}$ & $(\mathrm{C} 14: 0)$ & DPD & 1600 & 40000 & 275.4 & 275.4 & $1.1-5.4$ \\
\hline cool_C17_DW & $h_{3}\left(t_{5}\right)_{2}$ & $(\mathrm{C} 17: 0)$ & DPD & 400 & 10000 & 329.8 & 275.4 & $1.1-5.4$ \\
\hline heat_C17_DW & $h_{3}\left(t_{5}\right)_{2}$ & $(\mathrm{C} 17: 0)$ & DPD & 400 & 10000 & 275.4 & 329.8 & $1.1-5.4$ \\
\hline cool_C20_DW & $h_{3}\left(t_{6}\right)_{2}$ & $(\mathrm{C} 20: 0)$ & DPD & 400 & 10000 & 329.8 & 275.4 & $1.1-5.4$ \\
\hline heat_C20_DW & $h_{3}\left(t_{6}\right)_{2}$ & $(\mathrm{C} 20: 0)$ & DPD & 400 & 10000 & 275.4 & 329.8 & $1.1-5.4$ \\
\hline cool_C23_DW & $h_{3}\left(t_{7}\right)_{2}$ & $(\mathrm{C} 23: 0)$ & DPD & 400 & 10000 & 340.7 & 275.4 & $1.1-5.4$ \\
\hline heat_C23_DW & $h_{3}\left(t_{7}\right)_{2}$ & $(\mathrm{C} 23: 0)$ & DPD & 400 & 10000 & 275.4 & 340.7 & $1.1-5.4$ \\
\hline cool_DW & N/A & & DPD & 0 & 400 & 340.7 & 268.9 & $1.1-5.4$ \\
\hline \multicolumn{9}{|c|}{ MARTINI Simulations ${ }^{f}$} \\
\hline keyword $^{a}$ & \multicolumn{2}{|c|}{$\operatorname{lipid}^{b}$} & W type ${ }^{c}$ & $N_{\text {lip }}{ }^{d}$ & $N_{\text {wat }}{ }^{d}$ & $T_{\text {start }}^{e}(\mathrm{~K})$ & $T_{\text {end }}^{e}(\mathrm{~K})$ & $|\Delta T|^{e}(\mathrm{~K})$ \\
\hline cool_C12_W & DLPC & $(\mathrm{C} 12: 0)$ & $\mathrm{W}$ & 512 & 9728 & 300 & 265 & 1 \\
\hline cool_C12_PW & DLPC & $(\mathrm{C} 12: 0)$ & PW & 512 & 9728 & 300 & 265 & 1 \\
\hline cool_C16_W & DPPC & $(\mathrm{C} 16: 0)$ & $\mathrm{W}$ & 512 & 9728 & 325 & 273 & 1 \\
\hline heat_C16_W & DPPC & $(\mathrm{C} 16: 0)$ & $\mathrm{W}$ & 512 & 9728 & 273 & 325 & 1 \\
\hline cool_C16_PW & DPPC & $(\mathrm{C} 16: 0)$ & PW & 512 & 9728 & 325 & 273 & 1 \\
\hline heat_C16_PW & DPPC & $(\mathrm{C} 16: 0)$ & PW & 512 & 9728 & 273 & 325 & 1 \\
\hline cool_C16_PW & DPPC & $(\mathrm{C} 16: 0)$ & PW & 2048 & 38912 & 290 & 281 & 1 \\
\hline cool_C18_W & DSPC & $(\mathrm{C} 18: 0)$ & $\mathrm{W}$ & 512 & 9728 & 338 & 273 & 1 \\
\hline cool_C18_PW & DSPC & $(\mathrm{C} 18: 0)$ & PW & 512 & 9728 & 338 & 273 & 1 \\
\hline cool_W & $\mathrm{N} / \mathrm{A}$ & & $\mathrm{W}$ & 0 & 2128 & 338 & 265 & 1 \\
\hline cool_PW & N/A & & PW & 0 & 1824 & 338 & 265 & 1 \\
\hline
\end{tabular}

a "keyword" gives basic notation for each simulation, often used in figure legends. "lipid" describes the simulated lipid via standard DPD or MARTINI notation as well as the number of carbons in the saturated tails. "W type" describes the type of water bead used. ${ }^{d}$ In the various systems, the number of lipids $\left(N_{\text {lip }}\right)$ and water beads $\left(N_{\mathrm{w}}\right)$ are set so that their ratio is roughly equivalent for the two models, accounting for the different values of $n_{\text {mol }}$ for the water beads. ${ }^{e} T_{\text {start }} T_{\text {end }}$ and $\Delta T$ indicate the starting and ending points of temperature traces as well as the temperature discretization for each model. The values for DPD are noninteger due to the temperature conversion from the reduced units of DPD as described in the Supporting Information. ${ }^{f}$ Similar parameters and coding are also used for MARTINI simulations with $10 \%$ antifreeze water beads (WF), with results shown in the Supporting Information.

electrostatics in a nonuniform geometry should be considered with caution in general. ${ }^{45}$ However, in developing the polarizable water model, Yesylevskyy et al. found that both spherical truncation and particle mesh Ewald for the long-ranged interactions led to quite similar results for quantities such as area per lipid. $^{44}$

All simulations using the MARTINI model were performed with GROMACS 4.0.7. ${ }^{48}$ Analysis tools in GROMACS 4 were used to extract and calculate properties from the simulations. Calculation of the second-rank order parameter for the lipid tail, denoted here as $S_{\text {tail }}$ and defined in the Results section, was done using a script from the MARTINI Web site, ${ }^{54}$ with appropriate modifications for calculating the order parameter for all lipid tail lengths used in this study.

\section{RESULTS}

Below we present our results for each model independently. In the subsequent Discussion, we then compare the results between models with a more detailed perspective. Table 1 summarizes the simulations conducted for each model. For each lipid simulated, the basic suite of simulations conducted includes the following:

- a standard cooling run where the temperature is incrementally decreased

- often a subsequent heating run where the temperature is again increased to evaluate the hysteresis in the transition

- occasional runs with larger simulation boxes to assess finite size effects

For DPD, we consider four symmetric saturated lipids composed of three head beads $h$ and two tails of differing numbers of tail beads $t: h_{3}\left(t_{4}\right)_{2}, h_{3}\left(t_{5}\right)_{2}, h_{3}\left(t_{6}\right)_{2}$, and $h_{3}\left(t_{7}\right)_{2}$. Connecting $h_{3}\left(t_{4}\right)_{2}$ with DMPC as done in ref 15 and assigning three methyl(ene) groups per additional bead, this set of lipids corresponds to simulating tail lengths ranging from 14 carbons per saturated tail up to 23 carbons per tail. We shall employ the standard notation $\mathrm{Cn}_{\mathrm{C}}: 0$ with the first number indicating the number of carbons in the fatty acid chain and the second number indicating zero double bonds. For MARTINI, we simulate three different symmetric saturated lipids - DLPC (C12:0), DPPC (C16:0), and DSPC (C18:0) — based on the typical mappings for MARTINI. ${ }^{7,10}$ 


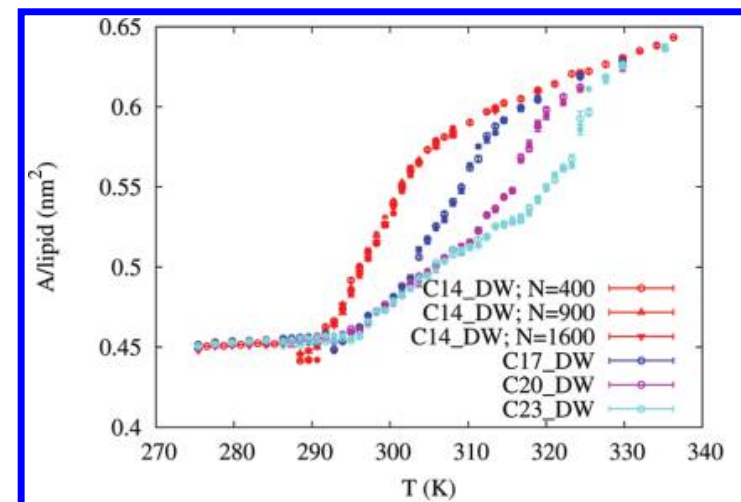

(a) $A_{\text {lip }}$

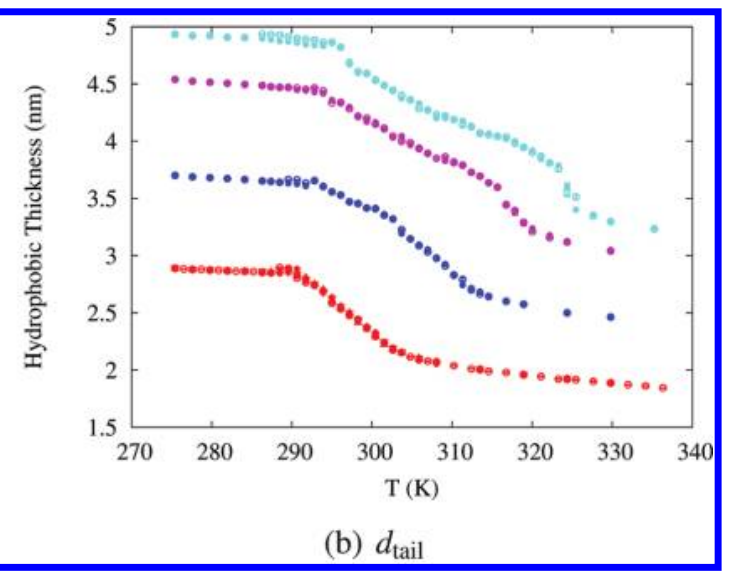

Figure 5. Area per lipid and bilayer hydrophobic thickness for the DPD model as a function of temperature for a variety of saturated lipid tail lengths and system sizes. Legend in (a) applies for both plots. Open symbols are for the cooling curves, and closed symbols are for the corresponding heating curves.

The fact that we simulate lipids with several different tail lengths is an important feature of our analysis. As DPD maps three methyl(ene) groups to one coarse-grained tail bead and MARTINI maps four groups to one tail bead, there is no single mapping from one coarse-grained lipid tail length back to a specific lipid. For this reason, all of our simulated data effectively have error bars along $n_{\mathrm{C}}$ of approximately \pm 2 . Thus, it is far more instructive to look at trends over varying tail lengths rather than the ability of either model to agree with experimental data for one single lipid tail length.

In all instances, we simulate bilayers in excess water of $75-76$ water molecules per lipid, a ratio well beyond the degree of hydration observed in multilamellar vesicles for fluid $\left(\mathrm{L}_{\alpha}\right)$ bilayers and the even less hydrated gel $\left(\mathrm{L}_{\beta^{\prime}}\right)$ bilayers. ${ }^{32}$ Previous simulations of the MARTINI model have shown the liquid-gel transition to be invariant to an increase in hydration beyond the experimentally observed hydration level. ${ }^{21}$ By simulating with such excess water, we aim to be guaranteed to simulate individual uncoupled bilayers separated by bulk water. Certainly transition temperatures change upon water-lipid composition, but here we consider only fully hydrated data. ${ }^{20}$

DPD Model. The phase behavior of saturated lipids with tails ranging from 14 carbons up to 23 carbons was studied with a cooling and subsequent heating loop for 400 lipids. In order to understand the extent to which finite-size effects are at play, DMPC (C14:0) is simulated with 900 lipids and 1600 lipids as well. Temperature is decremented in steps of $5.4 \mathrm{~K}$, with smaller temperature steps of 2.2 or $1.1 \mathrm{~K}$ in regions of rapid change of structural parameters. At each temperature, we simulated for a total of $1.4 \times 10^{5}$ Monte Carlo cycles. After an initial run of $2 \times 10^{4} \mathrm{MC}$ cycles containing approximately $3 \times 10^{5}$ DPD steps as well as 8000 attempted Monte Carlo moves to maintain $\gamma$ and $P_{\perp}$, an equilibration run at the subseqent temperature and a collection run at the current temperature are initiated. Typically all remaining $1.2 \times 10^{5} \mathrm{MC}$ cycles, with approximately $1.8 \times 10^{6}$ DPD steps and $4.8 \times 10^{4} \mathrm{MC}$ surface tension and pressure moves, contribute to equilibrium averages as the temperature is slowly decremented or incremented in the vicinity of transitions; however, in a few instances only smaller portions of the data are included in the equilibrium averages. Considering only the time evolution in the DPD steps, this effectively allows for $15.3 \mathrm{~ns}$ of equilibration and $91.8 \mathrm{~ns}$ of data collection. However, the MC moves in lateral area and volume certainly increase the effective time sampled and furthermore the soft interactions between beads lead to fast equilibration in particular in the solid phases. Rather than relying simply on these effective times, we carefully monitor our results for equilibration and sufficient sampling in area, volume, and ordering.

Structural Parameters. As has been previously demonstrated for varying lipid tail length, ${ }^{11}$ though in far less resolution, structural properties such as bilayer area and the hydrophobic thickness (defined as the average distance between the first beads of the tails in each leaflet normal to the bilayer) show signatures of transition as seen in Figure 5 for the subtransition, the pretransition, and the main transition, with the most substantial changes seemingly associated with the main transition. The order parameter associated with lipid tail tilt, $S_{\text {tilt, }}$ shows even stronger signatures of all transitions, as seen in Figure 6. We define this parameter as $S_{\text {tilt }} \equiv\left\langle(1 / 2)\left(3 \cos ^{2} \theta_{\text {tilt }}-1\right)\right\rangle$, where $\theta_{\text {tilt }}$ is the angle between the bilayer normal and the vector between the first and last beads of each lipid tail.

The peak-to-peak distance shown in Figure 7, defined as the distance between the most probable locations of the second head bead, also shows strong signatures for most of these transitions. Perhaps the most dramatic and distinctive signature in this peak-to-peak distance is that associated with the main transition. We caution the reader however that this sharp transition is simply pinpointing the temperature at which the distribution between liquid-like lipids and gel-like lipids in the DPD model ripple phase passes through the $50 \%-50 \%$ arrangement. ${ }^{11}$ In effect, then, this sharp jump in the peak-to-peak distance is a particularly straightforward way to identify the inflection point in area per lipid as a function of temperature. However, signatures of "bubbles" of either liquid at the lower temperatures or gel at the higher temperatures are evident in the entire range of $\mathrm{P}_{\beta^{\prime}}$ between the two dashed vertical lines in Figures 6 and 7. Therefore, while the main transition yields the most dramatic changes in area per lipid and hydrophobic thickness, identification of this transition is the most ambigous.

There are remarkably few effects of hysteresis in our simulations of the various lipids. Furthermore, finite size effects are barely present for our simulations of the lipid with C14:0 tails. The low temperature transition which we shall identify as the 


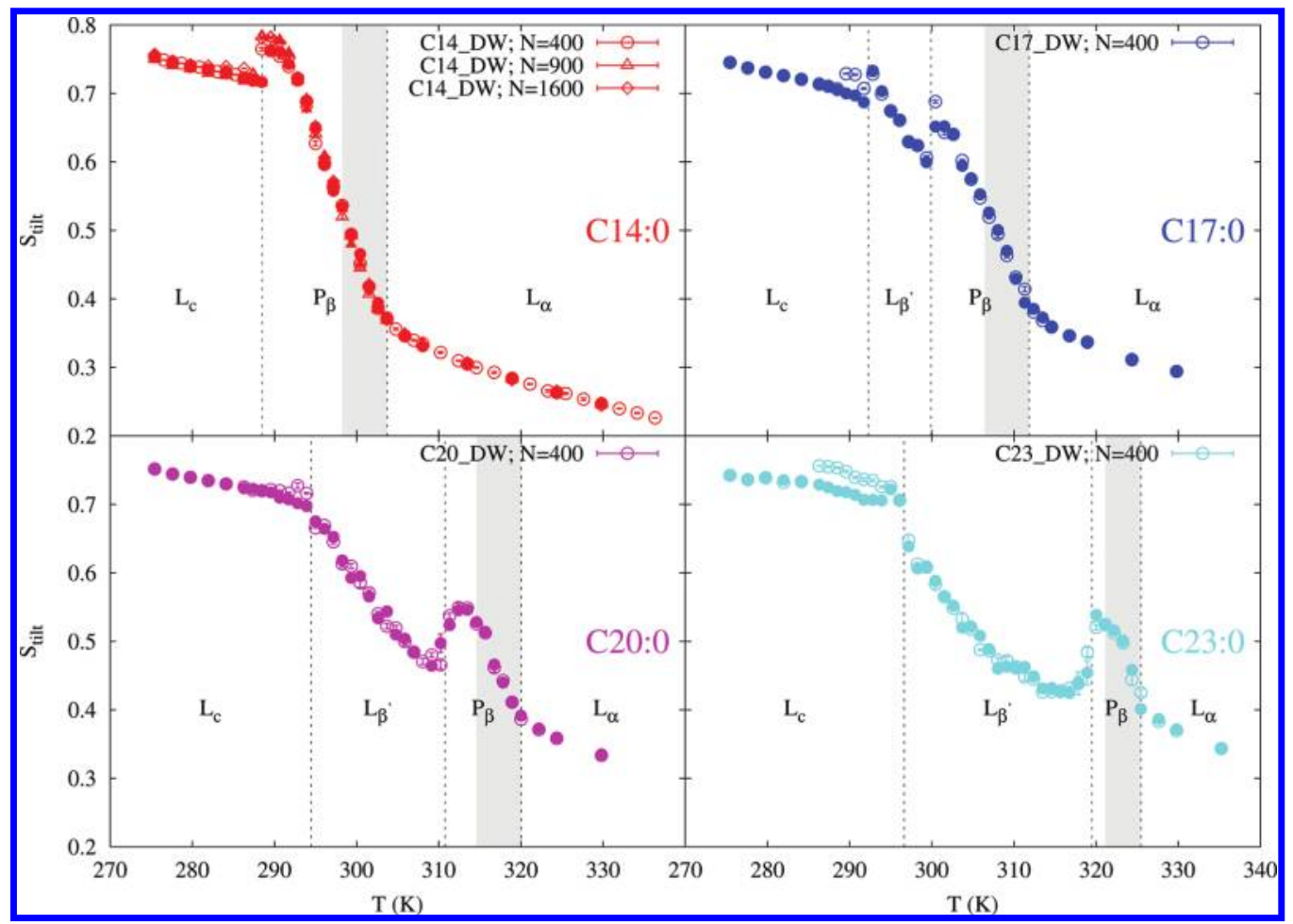

Figure 6. Lipid tail tilt order parameter $S_{\text {tilt }}$ for the DPD model as a function of temperature for a variety of saturated lipid tail lengths. The vertical dashed lines are meant to indicate the transition temperatures identified via structural and enthalpic characteristics. Each region between vertical lines is labeled with the identified phase: either $\mathrm{L}_{c}, \mathrm{~L}_{\beta}, \mathrm{P}_{\beta^{\prime}}$, or $\mathrm{L}_{\alpha}$. The shaded region indicates the temperature spread in identifying the main transition temperature between $\mathrm{P}_{\beta^{\prime}}$ and $\mathrm{L}_{\alpha}$.

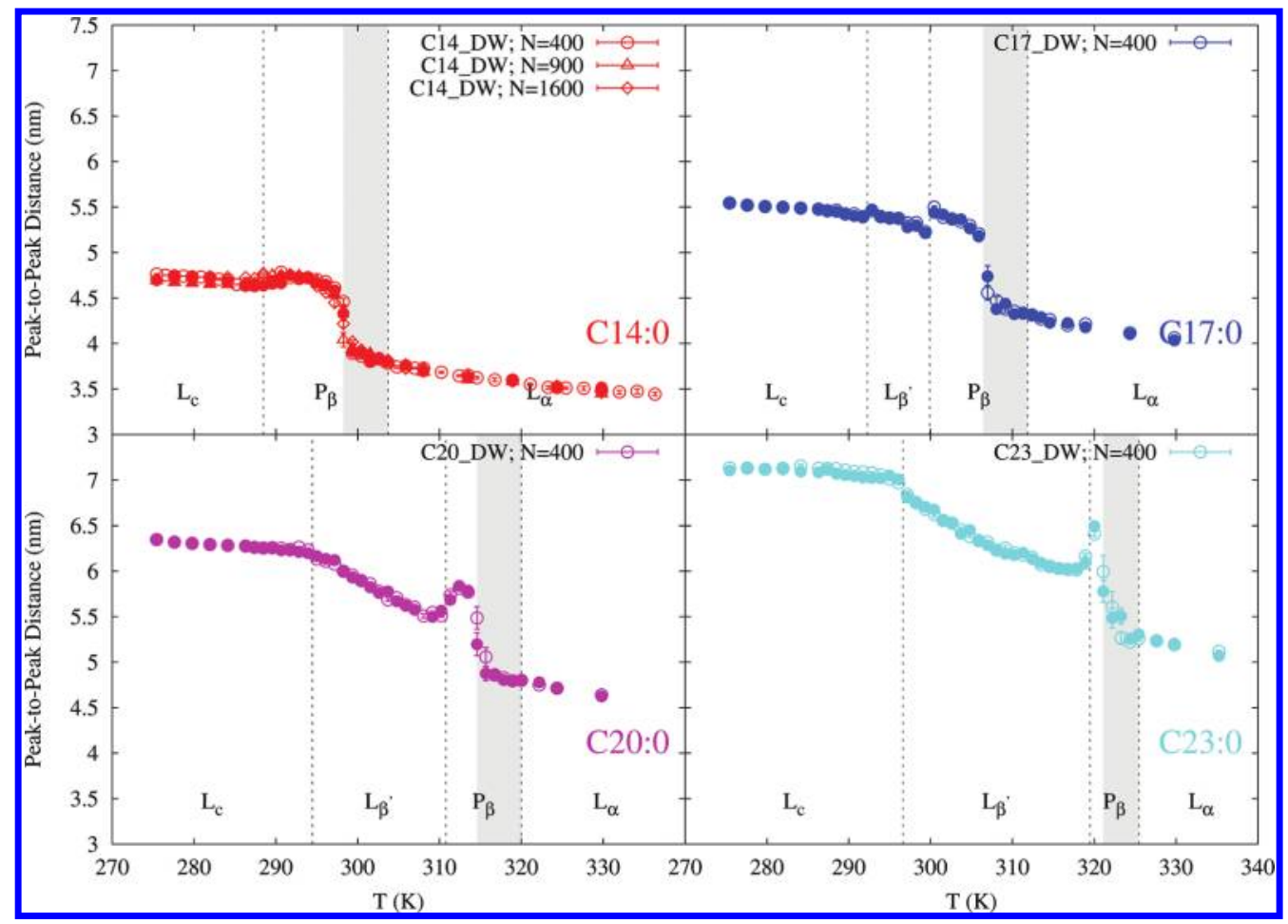

Figure 7. Peak-to-peak distance for the most probable distance between the phosphate beads for the DPD model as a function of temperature for a variety of saturated lipid tail lengths. Vertical lines as described in Figure 6.

subtransition has hysteresis of approximately $1 \mathrm{~K}$, and increasing the system size has no effect on the location of the transitions in DMPC (C14:0). There are some mild finite size effects associated with the extent of ordering in the subgel phase 


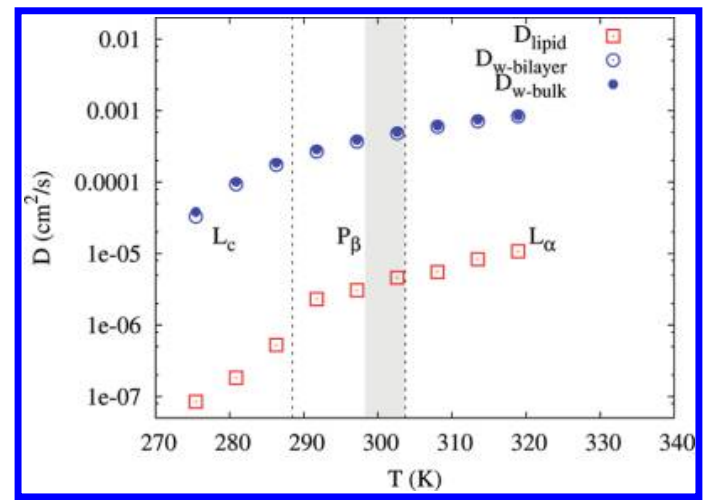

Figure 8. Calculated diffusion coefficients for the DPD bulk water and lipid bilayer models. The lipid examined with DPD is C14:0. Vertical lines as described in Figure 6, specifically for C14:0.

(see Supporting Information), but this has little to no effect on the location of the transitions.

Lipid Dynamics. We also examine the dynamics of the DPD model for bulk water, for the lipid C14:0 in a bilayer, and for the water solvating that bilayer. In each case, we calculate a coarsegrained diffusion coefficient $D_{\mathrm{CG}}$ by determining the slope of a line fit to $\left\langle R^{2}\right\rangle / 2 d$ as a function of time. The numerator $\left\langle R^{2}\right\rangle$ is either the mean square displacement of a water bead in three dimensions for bulk water or the mean square displacement of a lipid molecule or of a water bead in the two lateral dimensions of the bilayer, and $d$ is the dimensionality of the diffusive motion. We solely examine the lateral diffusion of water in the bilayer simulation, as the bilayer confines the motion of the solvating water in the direction normal to the bilayer. Following Groot ${ }^{57}$ and in accord with the MARTINI protocol for determining water diffusion, ${ }^{44}$ we convert this diffusion coefficient to physical units as

$$
D_{\text {phys }}=n_{\text {mol }} D_{\mathrm{CG}} \frac{l_{\text {red }}^{2}}{t_{\text {red }}}
$$

We use the reduced length and time units defined for DPD in the Supporting Information. The prefactor $n_{\mathrm{mol}}$ is required to account for the fact that the water bead is in fact composed of three different water molecules and the mean square displacement of a water bead will be a factor of $n_{\text {mol }}$ smaller than the mean square displacement of any individual constituent water molecule. $^{57}$

Shown in Figure 8 are the calculated diffusion coefficients (in physical units) for bulk water, for DMPC (C14:0) in a bilayer, and for the water solvating that bilayer. In order to calculate diffusion coefficients with DPD, we must run additional simulations beyond those run for our quasistatic heating and cooling curves insteading using NVT conditions as explained under Methods: Simulation Techniques. As such, we do not conduct an exhaustive study; rather we collect sufficient data to understand basic trends in dynamics and to have order of magnitude comparisons to experimental values.

Using our defined reduced units for time and length, we find that both water diffusion and lipid diffusion are substantially faster than experimental values. Relative to the experimental value of $2.3 \times 10^{-5} \mathrm{~cm}^{2} \cdot \mathrm{s}^{-1}$ at $300 \mathrm{~K}$, DPD bulk water diffuses over 21 times faster. Lateral diffusion for water solvating a bilayer is slowed slightly due to the coupling of the water solvation layer with the bilayer heads. For DMPC in a bilayer,
Almeida et al. found a diffusion coefficient of $6 \times 10^{-8} \pm 1 \times$ $10^{-8} \mathrm{~cm}^{2} \cdot \mathrm{s}^{-1}$ at $303 \mathrm{~K}$ using fluorescence recovery after photobleaching, ${ }^{58}$ and DPD C14:0 diffuses over 76 times faster. Lipid diffusion in the DPD model slows by a factor of 4.4 as the lipids gel at $T_{s}$, far less than the several orders of magnitude expected for such a transition. The consequences of this dramatically faster diffusion are explored in the Discussion.

Thermodynamics. Examining $H_{\text {lip }}$ in Figure 9, we see that, in contrast to the structural signatures previously displayed, only the subtransition exhibits a jump in the full lipid enthalpy. The main transition, which is readily identifiable from structural features in the DPD model with a moderately ambiguous position, has no apparent enthalpic signature. The pretransition, when it is not colocated with the subtransition, is also not identifiable. If we instead study the pseudoenthalpy $H_{\text {tail, }}$ all three transitions exhibit signatures of some form. However, even for this pseudoenthalpy, the main transition does not display a jump in $H_{\text {tail }}$. As such, we shall identify two transition temperatures for the main transition, one based on the sharp jump in the peak-to-peak distance, and the other associated with the disappearance of "bubbles" of alternate phases and roughly corresponding to the end of the "bump" in $H_{\text {tail }}$. The shaded region in each of Figures 6-9 graphically spans the space between these two possible identifications of $T_{\mathrm{m}}$, with the dashed line present at the temperature at which the "bubbles" of alternate phases disappear.

MARTINI Model. The behavior of the MARTINI bilayers were also studied by cooling the systems from the fluid phase. The simulations were started well above the main phase transition temperatures for each bilayer system, with DSPC, DPPC, and DLPC starting at 338, 325, and $300 \mathrm{~K}$, respectively. The systems were simulated for $625 \mathrm{~ns}$ at the target temperature. As is commonly done for MARTINI, we rescale these times by a factor of 4 , resulting in an effective time of $2.5 \mu \mathrm{s}^{*}$, with the asterisk denoting that it is a rescaled, effective time. Following the full simulation at the target temperature, the temperature was decremented by $1 \mathrm{~K}$ and a new simulation was performed at the new temperature target, corresponding to quasistatic cooling.

The DSPC and DPPC systems were cooled to $273 \mathrm{~K}$, the experimental freezing temperature of water. The DLPC system was cooled to $265 \mathrm{~K}$, since its transition temperature is determined experimentally to be at $273 \mathrm{~K}$. Only the last $500 \mathrm{~ns}^{*}$ at each temperature has been used for statistics, ensuring an equilibrated system. In a few cases when equilibration took slightly longer, less data has been used for statistics. The systems were composed of 512 lipids, divided evenly between the two bilayer leaflets. Each bilayer was simulated using two different water solvent representations: (a) MARTINI water beads (W) and (b) polarizable MARTINI water beads (PW).

The MARTINI water models are known to have bulk freezing temperatures above $273 \mathrm{~K}: 290 \pm 5 \mathrm{~K}$ for the original water model and 280-285 $\mathrm{K}$ for the polarizable water model. ${ }^{44}$ Our measured diffusion coefficients indicate that only $\mathrm{W}$ freezes in our simulations at $268 \mathrm{~K}$ while PW remains liquid. Further examination of the water density profiles for the polarizable water model supports the conclusion that freezing has not occurred in the PW simulations. However, bulk region water in our membrane simulations is already significantly ordered in the original $\mathrm{W}$ simulations at $275 \mathrm{~K}$. Therefore, simulations of the three lipid systems have also been performed with a mixture of $90 \%$ MARTINI water beads $+10 \%$ antifreeze water beads (WF) to avoid potential artifacts from water supercooling or freezing. 


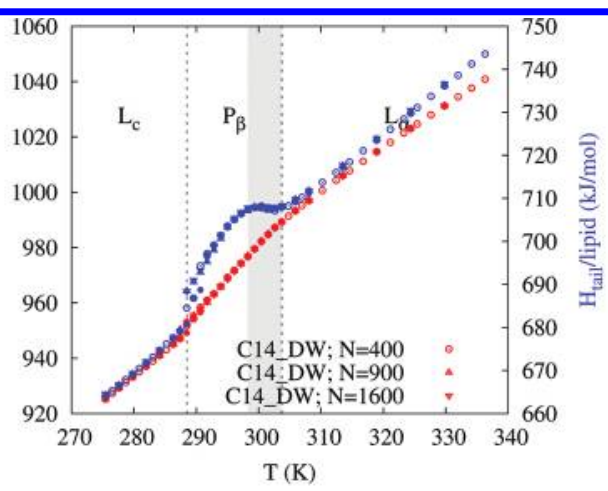

(a) $\mathrm{C} 14: 0$

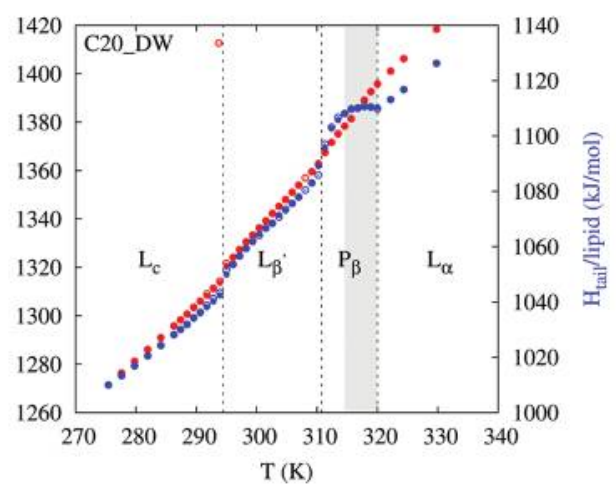

(c) $\mathrm{C} 20: 0$

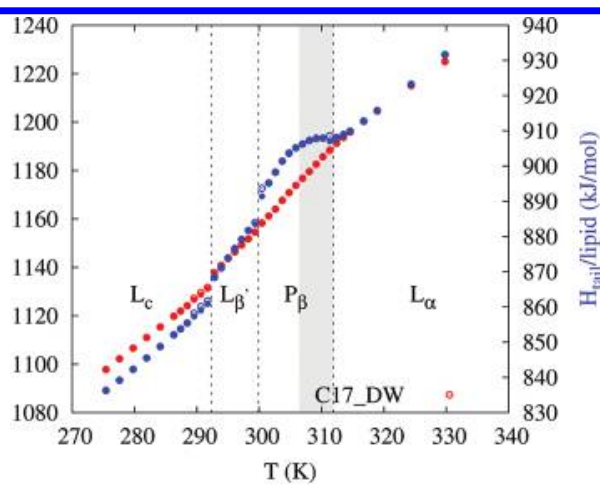

(b) $\mathrm{C} 17: 0$

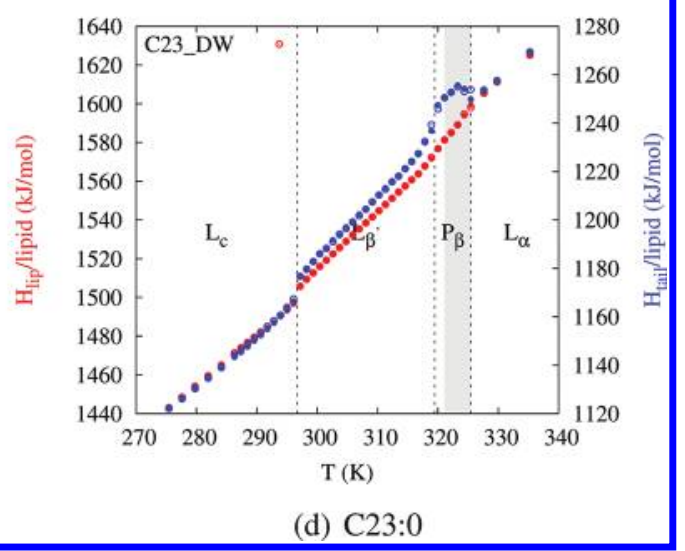

Figure 9. $H_{\text {lip }}$ and $H_{\text {tail }}$ for the DPD model as a function of temperature for a variety of saturated lipid tail lengths. $H_{\text {lip }}$ is displayed in red and associated with the left axis. $H_{\text {tail }}$ is in blue and connected to the right axis. Vertical lines as described in Figure 6.

The results of the latter simulations are similar to those of MARTINI water beads; therefore, for clarity, the results from these simulations are not included in the analyses below, but the data can be found in the Supporting Information.

Structural Parameters. As can be seen in Figure 10, various structural parameters exhibit signs of a phase transition for all three lipids studied. Specifically, we examine area per lipid in Figure 10a, bilayer thickness in Figure 10b, and the tail order parameter $S_{\text {tail }}$ in Figure 10c. Bilayer thickness is measured as the distance between the locations of the peak density of the phosphate beads in each leaflet normal to the bilayer. Similar to $S_{\text {tilt }}, S_{\text {tail }}$ is defined as $S_{\text {tail }} \equiv(1 / 2)\left\langle 3 \cos ^{2} \theta_{\text {bond }}-1\right\rangle$, where $\theta_{\text {bond }}$ is the angle between each individual bond within the lipid tails and the bilayer normal. While this $S_{\text {tail }}$ is distinct from the NMR order parameter, a correspondence between the behavior of this parameter and that of the NMR order parameter associated with the orientation of $\mathrm{C}-\mathrm{H}$ bonds has been shown for an all-atom bilayer model. ${ }^{15}$

For DSPC using the normal water model the transition initiates at $294 \mathrm{~K}$; however, an islet of disordered lipids persists for another couple of degrees and the transformation is not complete until $291 \mathrm{~K}$. Using the polarizable water model, the transition for the DSPC bilayer occurs at $293 \mathrm{~K}$. For DPPC the transition occurs at $286 \mathrm{~K}$ using the normal water model, whereas with the polarizable water model the initial transition occurs at $288 \mathrm{~K}$, but the phase transition does not progress to the same low state as the other systems. Instead an intermediate phase exists between 288 and $284 \mathrm{~K}$, whereafter the transition proceeds to the same level as the other systems. For DLPC, the transition occurs at $274 \mathrm{~K}$ using either water model. For the DLPC simulation with the normal water model, another transition occurs at $267 \mathrm{~K}$, which is freezing of water and is thus not related to the lipid phase transition, although it does affect the area per lipid.

To estimate the degree of hysteresis in the simulations, heating simulations have been performed for the DPPC systems starting from the systems after they were cooled to $273 \mathrm{~K}$. As can be seen in Figure 10d, the level of hysteresis is significant. When heating, the transition occurs at 312 and $311 \mathrm{~K}$, respectively, in the systems with normal and polarized water beads. The difference between cooling and heating is thus around $20-25 \mathrm{~K}$. The melting temperature is similar to that from earlier reports by Marrink et al. using instantaneous heating to $310 \mathrm{~K}$ from the gel phase. ${ }^{21}$

The area per lipid varies more strongly with temperature for the liquid phase than for the gel phase for all lipids. DSPC occupies $0.67 \mathrm{~nm}^{2}$ per lipid at $338 \mathrm{~K}$, and both DSPC and DPPC occupy $0.63-0.64 \mathrm{~nm}^{2}$ per lipid at $323 \mathrm{~K}$. At $300 \mathrm{~K}$, all three lipids exhibit an area per lipid of approximately $0.60 \mathrm{~nm}^{2}$. Throughout the gel phase, all three lipids have an area per lipid of $0.46-0.48 \mathrm{~nm}^{2}$. These values are comparable to previously reported values using MARTINI 1.0 and experiments in the fluid phase. ${ }^{10,32}$ The area per lipid is very similar for the three different lipids at the same temperatures, provided that they are in the same phase. This suggests that the area per lipid is determined by the lateral packings of the headgroups and the tail beads and not by the total length of the lipid tails. Interestingly, there seem to be two different levels of packing in the gel phase based on the area per lipid using the different water models, where the systems utilizing the polarizable water model pack more and reach lower 


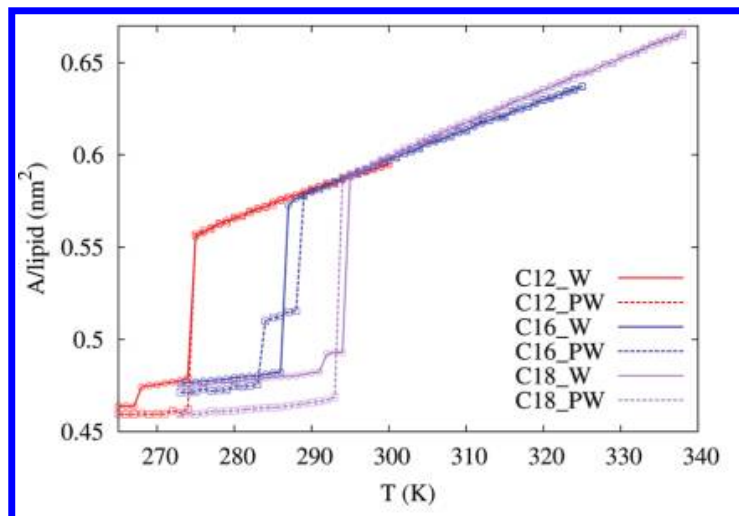

(a) Area per Lipid

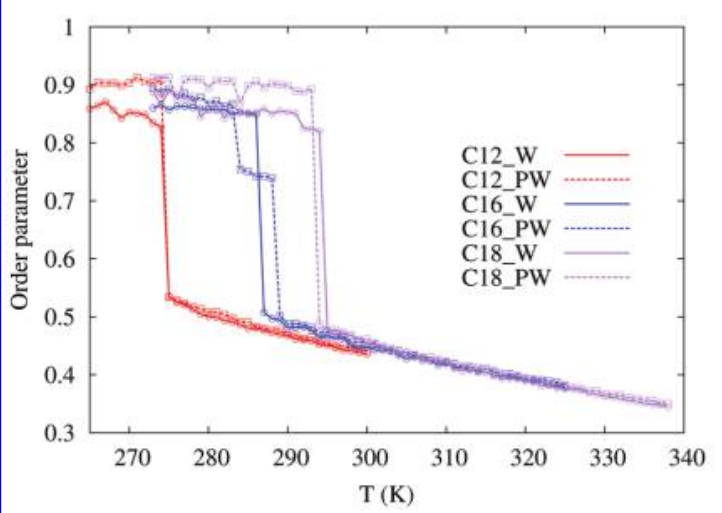

(c) $S_{\text {tail }}$

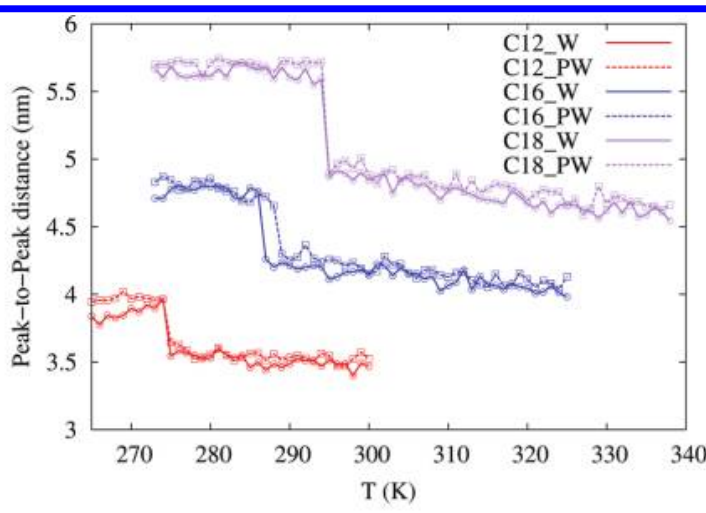

(b) Phosphate-to-Phosphate Distance

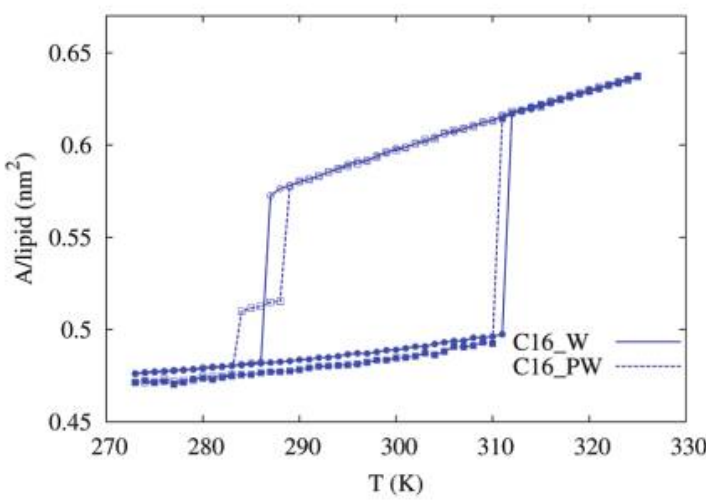

(d) Hysteresis in C16:0

Figure 10. Several structural parameters for the MARTINI bilayers. Part (a) displays the area per lipid for all three lipids with the two different waters on cooling, (b) shows the hydrophobic thickness, and (c) plots the tail order parameter for all six systems as a function of temperature. Finally, (d) shows the hysteresis present in the area per lipid for DPPC on heating.

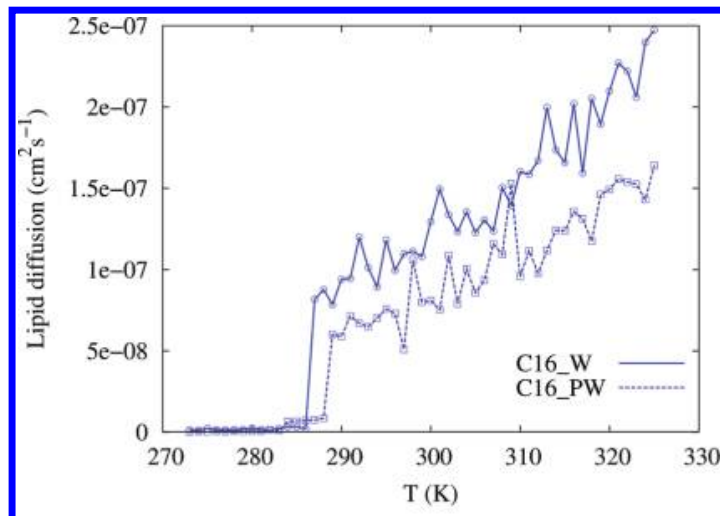

(a) DPPC Diffusion

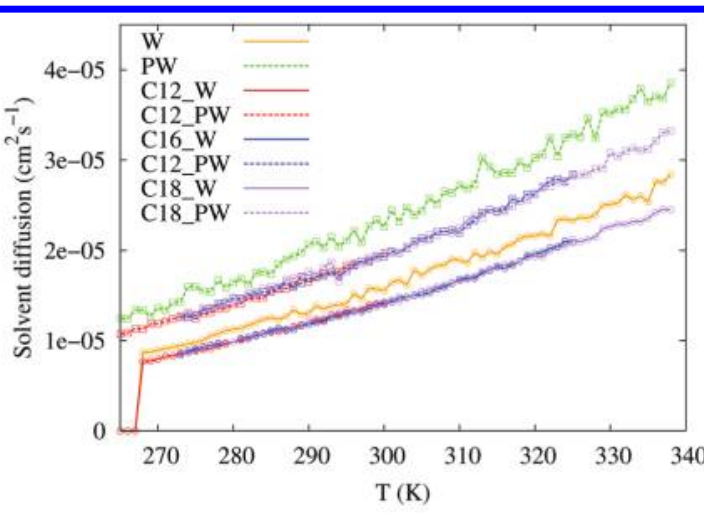

(b) W and PW Diffusion

Figure 11. Diffusion constant of DPPC as a function of temperature in (a), and diffusion constant of water as a function of temperature in bulk and with all lipids in (b) as a function of temperature for the MARTINI model.

areas per lipid. The same observation is readily apparent in the tail order parameter in Figure 10c reflecting tails more oriented along the bilayer normal in the gel phase and to a certain degree in the larger bilayer thickness in Figure 10b.

Lipid Dynamics. The lateral diffusion rate of the lipids in the bilayer is calculated in the same fashion as for DPD with time rescaled by $t_{\text {red }}=4 \mathrm{~s}$; for water, the only additional adjustment is that $n_{\text {mol }}$ is now 4 rather than 3 . The diffusion constant as a function of temperature for DPPC, shown in Figure 11a, with normal (polarizable) water varies over $(0.8-2.5) \times 10^{-7} \mathrm{~cm}^{2} \cdot \mathrm{s}^{-1}$ $\left((0.6-1.6) \times 10^{-7} \mathrm{~cm}^{2} \cdot \mathrm{s}^{-1}\right)$ as temperature is increased within the liquid phase. The lower mobility of the lipids in the presence of polarized water beads has previously been reported, and is likely a result of electrostatic friction between the lipid headgroups and water dipoles. ${ }^{44}$ Overall, these values for the lipid diffusion coefficient are in reasonable agreement with the experimental 


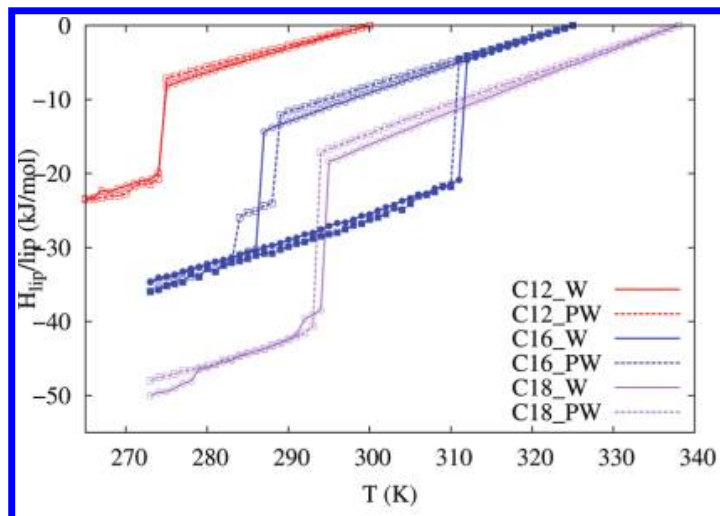

(a) $H_{\text {lip }}$

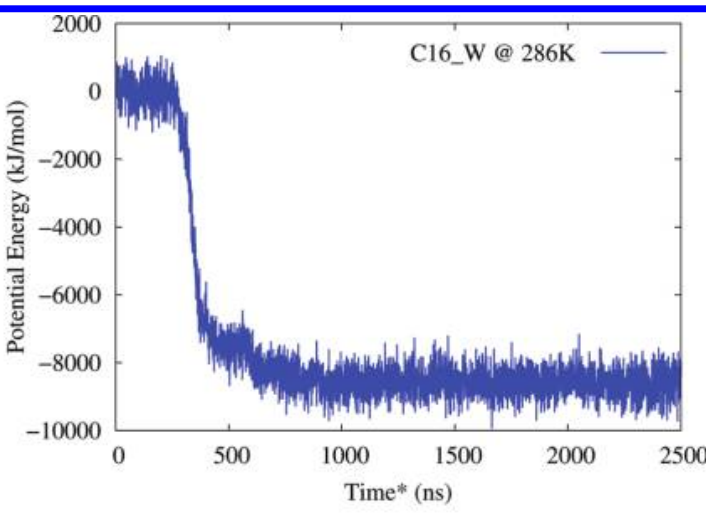

(b) $U(t)$ for DPPC at $286 \mathrm{~K}$

Figure 12. Thermodynamics of the phase transitions for MARTINI. In (a), $H_{\text {lip }}$ is shown for all lipid-solvent systems. In (b), the time trace of potential energy as a function of time for the cooling simulation of DPPC with W solvent at $286 \mathrm{~K}$ is shown.

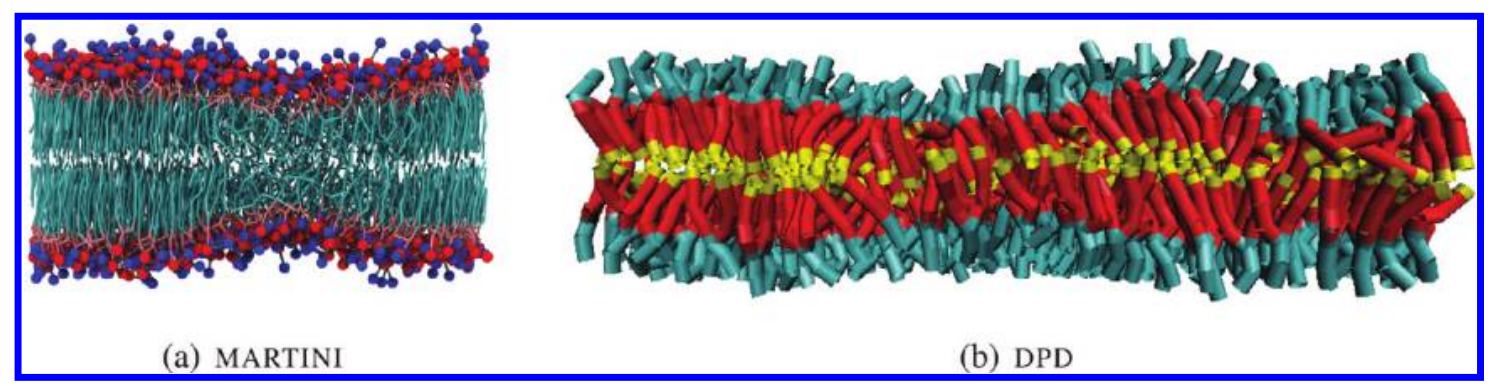

Figure 13. Snapshots of the observed "intermediate phase" for MARTINI DPPC with PW solvent beads in (a) and of the ripple phase seen in DPD in (b). The MARTINI bilayer consists of 512 C16:0 lipids. Seeming coexistence of gel-like lipids at the edges and liquid-like lipids in the center for MARTINI bear great similarity to signatures of the ripple phase explored in earlier work with the DPD model and shown in (b). ${ }^{11}$ The DPD bilayer is composed of 1600 C14:0 lipids. Colors for each model are as described in Figure 1.

value $^{58}$ of $6 \times 10^{-8} \pm 1 \times 10^{-8} \mathrm{~cm}^{2} \cdot \mathrm{s}^{-1}$ at $303 \mathrm{~K}$. In order to assess the diffusion coefficient of the lipids in the gel phase, longer simulations are required. We extended the cooling simulation of the DPPC bilayer at $275 \mathrm{~K}$ to a length of $10 \mu \mathrm{s}^{*}$. With this longer sampling time, the diffusion constant of the lipids was calculated to be $7.5 \times 10^{-10} \mathrm{~cm}^{2} \cdot \mathrm{s}^{-1}(2.5 \times$ $10^{-10} \mathrm{~cm}^{2} \cdot \mathrm{s}^{-1}$ ) for the systems containing normal (polarized) water. These values are similar to previously reported values for this model. ${ }^{21}$ The decreased lipid diffusion observed in both the fluid and gel phases in systems using polarized water beads is interesting in that the polarized water beads are in fact more mobile than the normal water molecules in the simulations, as shown in Figure 11b. As indicated in the area per lipid for the DLPC system using normal water beads, the diffusion calculations confirm that water freezes at $268 \mathrm{~K}$, leading to the further decrease in area per lipid at this temperature. This freezing is inconsequential in that it does not coincide with the lipid phase transition.

Thermodynamics. The transitions are observable in the various $H_{\text {lip }}$ profiles shown in Figure 12a, which reflect the observations from the structural and dynamical parameters. Excluding the intermediate phase observed for the DPPC system with polarized water beads and the islet of disordered lipids observed for DSPC with the normal water model, the transitions occur rapidly and no fluctuation between the two phases is observed, as shown in Figure $12 \mathrm{~b}$ for DPPC in normal MARTINI water at the transition temperature of $286 \mathrm{~K}$. There is a small dip in the enthalpy curve for DSPC at $278 \mathrm{~K}$, which is not reflected in all the structural features; however, the order parameter shows a slight increase here, which corresponds to a better packing of the lipids. The enthalpy change measured by DSC is an indicator of a phase transition. The change in $H_{\text {lip }}$ for using the normal water beads is 12,16 , and $22 \mathrm{~kJ} / \mathrm{mol}$ for DLPC, DPPC, and DSPC. Using the polarized water bead the values are 14,18 , and $24 \mathrm{~kJ} / \mathrm{mol}$, respectively. The interaction between the polarized water and the lipid head beads thus increases the change in enthalpy by $2 \mathrm{~kJ} / \mathrm{mol}$ for all three lipids.

Intermediate "Phase". The intermediate phase observed in the structural parameters (Figure $10 \mathrm{a}-\mathrm{c}$ ) and in the enthalpy (Figure 12a) for DPPC using the polarized water beads exhibits a seeming coexistence of the fluid and gel phases in the temperature interval 288-284 K, as shown in Figure 13a. During the simulation, exchange of the lipids between the phases is observed based on calculations of $S_{\text {tail }}$ parameter for each lipid during cooling from 288 to $284 \mathrm{~K}$. As this bears remarkable similarity to the presentation of the ripple phase in the DPD model as discussed in ref 11 , we sought to determine whether this phenomenon had similar stability with system size increase, which would then merit an identification as a distinct phase. As discussed carefully in the previous DPD work, for this to be a true ripple phase, it must not only remain stable at larger system sizes, but also maintain a similar characteristic length of gel-like and liquid-like domains leading to several wavelengths of the ripple, as shown in Figure 13b for 1600 lipids. In contrast to the DPD model, the intermediate phase could not be reproduced when starting the same calculation again from $292 \mathrm{~K}$ and cooling to $281 \mathrm{~K}$ or when switching to a larger system containg 2048 lipids in 
the same temperature range. This suggests that it is likely a result of kinetic trapping, although we are cooling at a reasonably slow rate.

\section{DISCUSSION}

In the following section, we seek to better compare the findings between the two models. First, we shall compare the basic structural features of the models. Subsequently, we shall discuss the dynamics in the two models both as they relate to questions of equilibration, hysteresis, and computational efficiency, and as they relate to the matching of energy and time scales for coarse-grained models. Finally, we shall address the use of both structural and our new thermodynamic computational benchmarks for identifying phase transitions in these models, as well as discuss what can and cannot be captured by both of the coarse-grained models we study.

In this discussion, we focus on the relative merits of each model for the quantities that we have studied in this paper. However, it is important to note that there are other important lipid properties not examined in this paper that each model would not be well chosen to represent. As one example, the DPD parameter set must be substantially modified in order to better match the rupture strain of the bilayer, but the consequences of this on the phase behavior studied in this paper are unknown. ${ }^{59}$ As another example, the MARTINI model does not even qualitatively capture the pressure versus area profile of a lipid monolayer. ${ }^{8}$

Comparison of Structural Features. As has previously been observed in a review of many different coarse-grained models of lipids, ${ }^{4}$ one remarkable feature of these results is the similarity between like phases in the two models. Within the uncertainty in mapping a coarse-grained representation onto a lipid of specific tail length, both DPD and MARTINI agree well with the experimental values for lipid area and hydrophobic or bilayer thickness, for both the fluid and the gel phases. As one example, for area per lipid, Nagle and Tristram-Nagle report that, for $\mathrm{DPPC}$, the area per lipid changes from $47.9 \AA^{2}$ in the gel at $293 \mathrm{~K}$ to $64 \AA^{2}$ in the liquid at $323 \mathrm{~K}$. While MARTINI has slightly better agreement with experiment for the absolute values of area per lipid once the gel phase is extrapolated to $323 \mathrm{~K}$, the $\Delta A=$ 16.1 $\AA$ is equally well described by both the DPD and MARTINI models. The DPD model C17:0 yields a shift from 45.5 to $62 \AA^{2}$ over that temperature difference, with $\Delta A=16.5 \AA$. The MARTINI model yields a shift from 47.5 to $63 \AA^{2}$ between the gel phase extrapolated to $293 \mathrm{~K}$ and the liquid phase at $323 \mathrm{~K}$, with $\Delta A=15.5 \AA$. For the DPD and MARTINI lipid models, many of these structural changes have been observed and studied before; ${ }^{10,11,21}$ however, the temperature resolution employed in the cooling and heating curves allows a numerically far more definitive determination of these structural boundaries than previously understood.

One point of contrast between the DPD and MARTINI models is that the DPD model has a tilted gel phase and a subgel phase, while the MARTINI model has a gel phase with tails predominantly oriented with the bilayer normal: results which have been reported for both models separately. ${ }^{11,21}$ These two observations may be cleanly reconciled by the observation that the area and tilt of the gel phase are determined by the balance between the effective lateral packing distances of the headgroup and the tail beads within one leaflet of the bilayer. If the effective packing distances of the headgroups are comparatively large, then the lipid tails may adopt the tilted orientation present in the triclinic crystal structure of the equivalent $n$-alkanes. ${ }^{60}$ If these

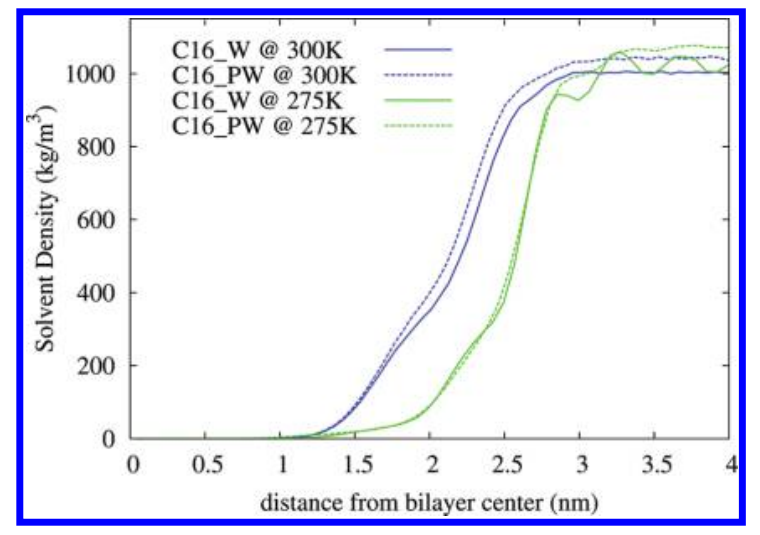

Figure 14. Water bead profiles for both the standard MARTINI water model and the polarizable MARTINI water model near a DPPC bilayer. Results are averaged over both bilayer leaflets with density profiles shown from the center of the bilayer at the left to the bulk solvent phase at the right. Profiles are shown for both the fluid phase at $300 \mathrm{~K}$ (blue) and the gel phase at $275 \mathrm{~K}$ (green). More dense solvent phases are evident for the polarizable water. Beyond this, the most substantial difference lies in the ordering and lipid head intercalation of the water beads in the gel phase.

distances are small, then the lipid tails are constrained to an untilted conformation in order to minimize contact between the unshielded hydrophobic tails and the solvent. For the DPD force field, these packing distances may be varied relatively directly by tuning the repulsion parameter between headgroups, and the lipid behavior follows the basic considerations described above. ${ }^{11}$ For the MARTINI model, previous work had found that a tilted gel phase could be reproduced at low levels of hydration by decreasing the lipid tail bead radius by $10 \%$ in the Lennard-Jones interactions, ${ }^{21}$ thereby tuning the relative lateral packing distances between headgroups and between tail beads. Headgroup lateral packing distances may not be tuned as directly in MARTINI as they are an effective property arising from both the Lennard-Jones and Coulomb interactions between headgroups as well as with water.

Interestingly, for the MARTINI model with the polarizable water bead, the packing of the lipid heads in the gel phase appears to be more laterally compact than that found with the unpolarizable water bead. Hypothesizing that this must be related to the ordering of the water beads at the interface with the charged beads of the phosphatidylcholine headgroups, we examined the water bead density for both water models in the DPPC system in the fluid phase at $300 \mathrm{~K}$ and in the gel phase at $275 \mathrm{~K}$. The closer head packing for the polarizable water bead might suggest that the water density in the head region should be lower; however, the density profiles shown in Figure 14 do not support this hypothesis. The standard MARTINI water beads are more ordered in the vicinity of the bilayer surface than the polarizable MARTINI water beads, likely a consequence of the fact that the MARTINI water beads are supercooled. However, the intercalated density of the polarizable water beads is higher, suggesting instead as a possible mechanism that the polarizable water beads mixed among the lipid headgroups mediate the interactions between like-charged headgroup beads, leading to effectively a greater headgroup attraction and smaller lateral packing distance. Certainly, the lack of water bead density oscillations seems more physical for a liquid phase; however, more careful examination of this effect with the long-ranged charge-charge interactions is merited. 
We also hypothesize that the adjustment for the tail bead volume required to reproduce a tilted gel phase with polarizable water might be more substantial as these results point to a lipid headgroup with a smaller lateral area.

Consequences of Dynamics in Models. Examining the dynamics in both of these models is a good example of coarsegrained models being unable to match all properties as well as of the trade-offs between efficient equilibrium sampling and accurate dynamics. The following discussion of various points of lack of correspondence gives us some insight into the basic reality of coarse-graining: it is not generally possible to capture all phenomena when averaging out many internal degrees of freedom. Rather the aim may be to reproduce a few chosen properties and seek to understand where the resulting model succeeds and fails in representing other properties. In the case of the models examined here, the coarse-graining was conducted with an eye toward matching certain equilibrium properties, rather than dynamic.

At the most basic level, neither the DPD model nor the MARTINI model was parametrized to match the dynamics of the lipid/water system. Therefore, a priori, there is no reason to expect correct collective dynamics to arise from these model parametrized equilibrium properties. In an extensive study of the basic DPD water model by Groot and Warren, the time scale was set by matching water diffusion., 97 The energy scale then directly results from the mass, length, and time units. However, in the extension of the DPD model to study saturated lipids, Smit and co-workers instead set the energy scale by mapping bilayer transition temperatures between the full system and the true system, meaning that the time scale is no longer free for unit matching since the energy scale implies a time scale as in molecular dynamics simulations of Lennard-Jones systems. ${ }^{11,15}$ Given that the relative time scales for diffusion between lipids in the gel phase and in the liquid phase in the DPD model differ by far less than the orders of magnitude observed experimentally, attempting to set our time and energy scales by dynamics matching would be fruitless. Therefore, our choice of setting the energy scale by transition temperatures as detailed in the Supporting Information is the more reasonable option. Furthermore, as one of the aims of DPD is to achieve the fastest dynamics possible while retaining the important driving forces of the system, this dramatically accelerated dynamics is expected and has consequences for the equilibrium sampling of the system.

In MARTINI, the energy scale is set by the process of model development and parametrization, matching the free energies of vaporization, hydration, and partitioning of various lipid building blocks. ${ }^{7}$ As such, the time scale is already set in the traditional molecular dynamics sense. However, phenomenologically, the MARTINI force-field developers found a remarkably similar effective scaling of time for water diffusion, lipid diffusion, water permeation though bilayers, and the aggregation of lipids into vesicles. All phenomena roughly match known time scales with an effective rescaling of time by a factor of $4 .^{7}$ This fortuitous correspondence has encouraged the extension of MARTINI simulations to the study of dynamics. As demonstrated here, the orders of magnitude slowdown of dynamics between the liquid and the gel phases is also captured by the MARTINI model.

These very basic results indicate that certainly MARTINI is a more appropriate force field than DPD for observing dynamical events. This very likely is a consequence of the more realistic representation of effective core repulsions coupled with attractions, especially as compared to the effective soft repulsions of the DPD model. However, our analysis of the solvent and lipid dynamics using the new polarizable water model in MARTINI indicates that caution must still be employed in interpreting these dynamics. With the new polarizable water bead, the dynamics of the water diffusion has in fact become faster while the lipid dynamics has conversely slowed. If the effective time scale were rescaled according to the water diffusion, the lipid diffusion would be even slower. While certainly the slowing effect of the solvent polarizability on the lipid motion is explainable, ${ }^{44}$ this differing effect of the polarizability on the water and on the lipids simply highlights the known caveat that this scaling of time by a factor of 4 for MARTINI is based in phenomenology and dynamical calculations must be interpreted with some caution. $^{7,10,54}$

The nonphysical accelerated dynamics in the DPD model has beneficial effects for the sampling of equilibrium structures. The comparatively fast dynamics, coupled of course with the accelerated sampling of area and volume fluctuations by the hybridization of DPD with MC moves, results in markedly few signs of hysteresis or finite size effects during these transitions. In order to better support this observation, we proceed to make a rough estimate of the acceleration of sampling for DPD as compared to that of MARTINI. We do not undertake a direct timing comparison of simulations as they are conducted via different codes, but rather make several reasonable scaling arguments. Before considering the accelerated diffusion, we first consider the number of interaction pairs calculated for each time step. The cutoff radius for force evaluation for DPD is $6.46 \AA$ while it is 12.0 A in MARTINI. Thus for each given coarse-grained bead, the DPD model requires evaluating pair forces in roughly onesixth of the volume. This would naively give DPD a factor of 6.4 speedup. However, as MARTINI beads represent four water molecules or methylene groups rather than the three groups per bead for DPD, this is reduced to a factor of 4.8 for each time step, for the nonpolarizable water model. This factor of 4.8 should be seen as an estimate, as there are other simulation implementation details that we have not assessed here. ${ }^{61}$

However, to assess computational efficiency for equilibration, we must evaluate the collective dynamics of the trajectory as a whole. The effective time step for DPD is $51 \mathrm{fs,}$, while for MARTINI the time step is $120 \mathrm{fs}^{*}$ (30 fs prior to time rescaling). This scales the computational advantage of DPD down to a factor of 2 . We must also account for the difference in effective diffusion rates. In the liquid phase, MARTINI lipids diffuse at roughly physical rate, while DPD lipids diffuse 75 times faster. Thus in sampling the liquid phase, DPD is about 150 times more efficient. The computational efficiency of DPD is even greater in the gel phase. These scaling arguments illustrate why DPD yields less hysteresis in transitions than MARTINI despite DPD dynamics sampling approximately $110 \mathrm{~ns}$ while MARTINI dynamics sampled $2.5 \mu \mathrm{s}^{*}$. The differences in computational efficiency due to MC sampling of volumes and bilayer areas in DPD-MC rather than Berendsen barostated sampling in MARTINI are more challenging to estimate; we simply note that the lack of hysteresis of our DPD results suggests that the MC sampling of cell shape may further accelerate equilibration.

This dramatic acceleration of sampling within the DPD model is a result of both the strongly accelerated dynamics outlined above and the smooth and slowly varying interactions between particles' cores leading to a substantially lower kinetic barrier to transition between the liquid and gel phases. In general, these observations about the sampling efficiency of the DPD potentials are not entirely novel. The preceding analysis yields insight 


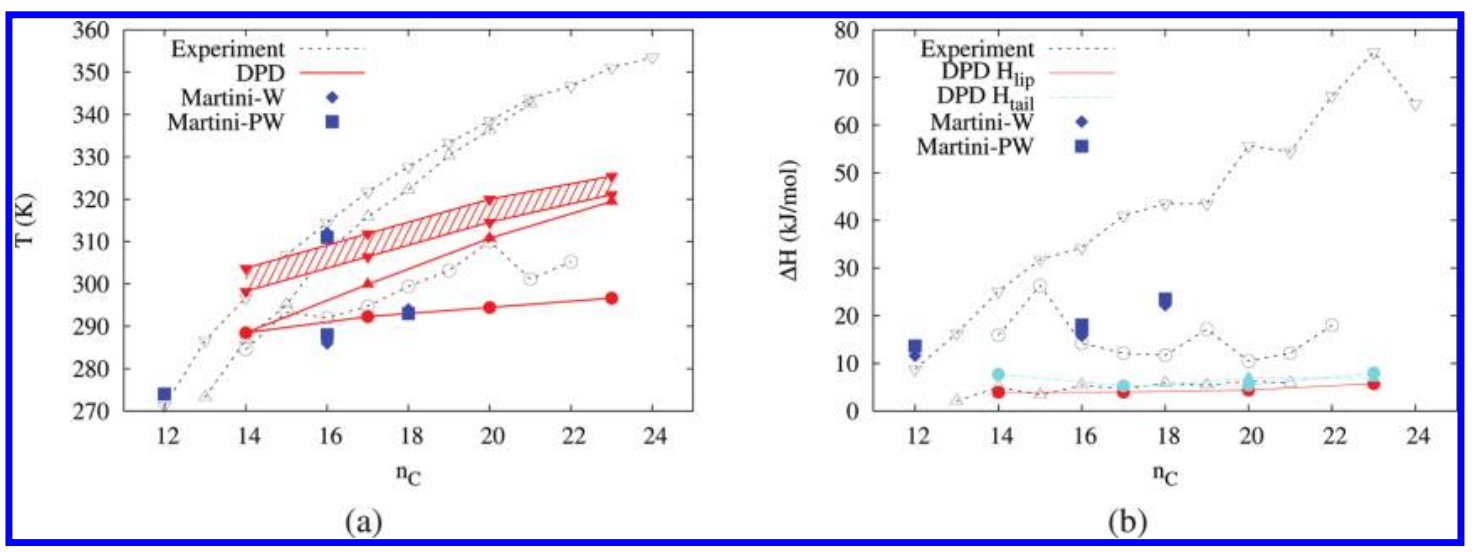

Figure 15. Comparison of experimental data from Koynova and Caffrey ${ }^{20}$ to results obtained from the DPD and MARTINI models. Symbol identities are as in Figure 3 with $\bigcirc$ indicating the subtransition, $\triangle$ indicating the pretransition, and $\nabla$ indicating the main transition. Open symbols are for the experimental results with thin dashed lines connecting the points to emphasize trends; the corresponding closed symbols are for the computational DPD results. The shaded region between the points for the main transition for DPD in (a) connects the two possible identifications of the main transition temperature. The DPD results appear in red, with the exception of $\Delta H$ determined via $H_{\text {tail }}$ which is in cyan. The MARTINI results are displayed in blue with two new symbols, as each MARTINI model displays only one transition. Results for both the cooling and heating curves for DPPC in MARTINI are displayed, with the uppermost point in (a) corresponding to the heating result.

similar to that found in ref 57 when comparing DPD to all-atom molecular simulation; however, as argued, these considerations are still present when comparing coarse-grained models with soft potentials to those with effectively hard cores. The soft potentials employed in DPD enable a more detailed exploration of parameter space as their equilibration is more efficient.

Characterization of Phases with Computational DSC. Before delving into the model results more closely, we first make several general observations. For these coarse-grained systems, we find that computational DSC does indicate a transition in each model that corresponds closely with an observed structural transition. In Figure 15, both (a) the transition temperatures found either via structure or calorimetry and (b) the enthalpy changes found via calorimetry are compared to the experimental values tabulated by Koynova and Caffrey. ${ }^{20}$ Both models have aspects of agreement and disagreement with the details of the experimental transition data, which we shall explore more completely in the following.

Overall, the best agreement we could hope for in a coarsegrained model is quantitatively matching the transition temperatures and qualitatively matching the relative scaling of $\Delta H_{\mathrm{m}}$ $\Delta H_{\mathrm{p}}$, and $\Delta H_{\mathrm{s}}$. Quantitative agreement of these latent heats is too much to expect from a coarse-grained model that was not explicitly developed to model these phase transitions. In coarsegraining, intramolecular degrees of freedom are necessarily lost and this almost certainly results in lower entropy changes and therefore lower enthalpy changes in a given phase transition.

The DPD lipid model is a remarkably simple coarse-grained model that presents the range of structural phases expected for single component saturated lipid bilayers as presented here and in ref 11 as well as the range of structural phases expected for bilayers that are binary mixtures of saturated lipids and cholesterol. ${ }^{15}$ This model does yield the full range of structural phases, the basic trends of transition temperatures with lipid tail lengths, and the eventual disappearance of $\mathrm{P}_{\beta^{\prime}}$ as the lipid tail length is increased, provided that this transition temperature is determined via the peak-to-peak distance. However, the transition temperatures which agree the best are those for DMPC (C14:0), with which the temperature and energy scales are set. The further trends in transition temperatures as a function of tail length agree less well, though the basic trends are there. This already hints that the energy scale for DPD is predominantly phenomenologically based, as will be more evident in the analysis of the enthalpies of transition. As the DPD model is purely repulsive and the parameters are given no temperature dependence, a phenomenological energy-scale mapping is the best that could be hoped for.

When studying the thermodynamics of these DPD transitions as revealed by $H_{\text {lip }}$, the transition with the most dramatic changes in structural features as well as the largest experimental $\Delta H$ $\left(\mathrm{P}_{\beta^{\prime}} \rightarrow \mathrm{L}_{\alpha}\right)$ has no enthalpic signal of a first-order transition attached to it. The transition which yields the smallest structural changes $\left(\mathrm{L}_{\mathrm{c}} \rightarrow \mathrm{L}_{\beta^{\prime}}\right)$ is the only transition with an observable enthalpic signature. Perhaps unsurprisingly, this transition is also the only one for DPD which has even small signatures typical of phase transitions such as hysteresis and finite size effects. Beyond this, the $\Delta H_{\text {lip }}$ associated with this transition is substantially smaller than the experimentally observed $\Delta H$ for any of the lipid phase transitions. However, given the highly coarse-grained DPD model with smoothed interactions and the total lack of attractive interactions, we should not reasonably expect the $\Delta H_{\text {lip }}$ values to be matched regardless.

Examination of $\Delta H_{\text {tail }}$ reveals signatures corresponding to the structural rearrangements for all observed structural changes; however, as noted, this is a pseudoenthalpy focusing on the lipid tails, but a net thermodynamic signal does not remain for $\Delta H_{\text {lip. }}$. One way to view these results is that the DPD model has only one true first-order transition and the other structural changes are not true "thermodynamic" first-order transitions, as the change in enthalpy for a phase transition as observed by DSC would seem to be a requisite signal. Alternatively, we argue that these results suggest we look at what effects might be missing in the DPD model that would lead to a stronger enthalpic signature for our observed structural changes. Two possible hypotheses might be the absence of actual attractions between coarse-grained sites and a lack of detail in the electrostatic coupling between the solvent and the zwitterionic lipid headgroups. In this sense, study of the MARTINI model with two different water models provides a way to investigate these claims. 
As previously found for the original MARTINI model, ${ }^{21}$ only one transition between the gel phase and the fluid phase is evident for both the standard MARTINI water model and the polarizable MARTINI water model even with quasistatic cooling and heating. When considering the correspondence between the calculated MARTINI transition temperatures with the experimental data, the transition temperatures during cooling appear to closely correspond with the subtransition while the transition temperature of DPPC during heating corresponds markedly well with the range of the pretransition and the main transition. As MARTINI has been shown to have hysteretic and finite size effects, this correspondence is not necessarily meaningful, but it is interesting to hypothesize that perhaps the other lipid bilayer phases are masked by the rather large hysteresis evident in the basic gel to fluid transition. One tantalizing hint of this is the ripple-like intermediate "phase" observed for DPPC with the polarizable water model, but this was not borne out with tests for reproducibility or stability as system size was increased. Given that the MARTINI model has been shown to display the structural signatures of a ripple phase with effectively smaller tail beads and low hydration, ${ }^{21}$ we conducted a preliminary examination of the phase behavior of this altered model at full hydration but found no evidence for the presence of other phases, neither rippled nor tilted.

The MARTINI model does yield better quantitative agreement with the typical magnitudes of $\Delta H$. The $\Delta H_{\text {lip }}$ found for MARTINI is quite similar to that of the subtransition experimentally. This better alignment of transition enthalpy magnitude is likely connected to MARTINI matching various solvation free energies during its parametrization protocols. Given that we might hypothesize that the transition captured by MARTINI is to a large extent an order-disorder transition in the lipid tails, it is challenging to dissect how these solvation free energies necessarily correlate with the transition enthalpies. However, alternatively, the contribution to the transition enthalpy captured may instead represent the different degrees of hydration of the lipid headgroups and lipid tails in these two phases, a free energy that was reasonably matched during the parametrization. To the degree that the MARTINI model also does not capture the full extent of enthalpic change from low temperature to high temperature, this suggests that we have hit the limits of this extent of general coarse-graining where some relevant degrees of freedom are necessarily lost. The full range of enthalpic change is likely due to the variation of degrees of freedom internal to a coarse-grained bead as a function of temperature. To fully describe such enthalpy changes at our given level of coarsegraining would likely require temperature-dependent parameters, a requirement which is clearly well beyond the parametrization conducted by MARTINI as well as beyond the scope of most coarse-graining studies. The extent to which MARTINI captures the magnitude of enthalpy changes is promising given that model was developed simply for simulations at ambient conditions.

One intriguing possibility for future work with the MARTINI model in analyzing the enthalpies of lipid phase transitions is conducting a study of the model with a smaller tail bead diameter. While our initial study via quasistatic cooling at complete hydration did not yield the ripple phase found in the hydrated system, ${ }^{21}$ it would be interesting to study what structural and thermodynamic signatures are evident as the relative tail bead and head bead sizes are varied. Such model tuning would lead to a more complex parameter space exploration for MARTINI parameter fitting, as typically the bead diameter is not varied. Further checks would also be necessary to remain consistent with the basic MARTINI methodology of setting the effective parameters by the solvation thermodynamics of various molecular building blocks. However, this tuning might lead to further insight into the relevant organizational forces in the MARTINI model. If, indeed, the inclusion of interbead attractions is most crucial for exhibiting enthalpic signatures for the main transition and the pretransition, then such a modified MARTINI model would have hopes of displaying such signatures. If, instead, the inclusion of internal bond degrees of freedom is necessary for these transitions, then the MARTINI model might exhibit effects similar to those for the DPD model where only the subtransition displays a change in enthalpy.

\section{- CONCLUSIONS}

We have compared two established coarse-grained models of the solvated lipid bilayer - DPD and MARTINI — for one of the simplest bilayer compositions exhibiting a phase transition: diacyl phosphatidylcholines. Our goal was twofold. First we sought to better characterize the extent to which these models capture the organizational driving forces for this simple bilayer system. Second, we introduced a new way to study these transitions, examining not just structural order parameters but also calculating a simple thermodynamic signature of the change measurable experimentally: $\Delta H_{\text {lip }}$. For bilayer simulations with explicit solvent, removing the bulk water contributions to the enthalpy does indeed lead to observable transitions in $H_{\text {lip }}$ that correspond to observed structural transitions.

Perhaps most striking is the mere fact that both MARTINI and DPD do reproduce important (and different) features of the phase diagram. The DPD model displays the full range of phases when considering the structural parameters, and one of these transitions is a true first-order transition as determined by $\Delta H_{\text {lip }}$. Furthermore, as previously found with less precision, ${ }^{11}$ this model captures the basic topology of the phase diagram as well. However, the enthalpy change associated with the subtransition between subgel and gel is substantially underestimated, and no observable enthalpy change is present for the structurally more significant transitions among the gel, ripple, and fluid phases. Possible reasons for this include (1) the very smooth soft repulsive interactions, (2) the lack of a balance between core repulsions and longer-ranged attractions, and (3) the lack of molecular internal degrees of freedom. Of course, these are exactly the model features which also lead to fast dynamics and a lack of kinetic barriers in the transition between observed structural regimes in the DPD simulations. Some of these inaccuracies for the purely repulsive model might be rectified by introducing temperature-dependent parameters; however, that again serves to destroy the simplicity of the model.

The MARTINI model appears less able to capture the range of phases based on our studies with "quasistatic" cooling and heating. Yet the model does exhibit substantial hysteresis between the liquid and gel phases, as expected for a first-order transition. This hysteresis may mask other underlying equilibrium phases. Future work might explore this question via equilibrium umbrella sampling along a relevant structural order parameter. Overall, enthalpies associated with the observed change are numerically more in line with experiments. As is already known, MARTINI is much more appropriate for dynamical studies. However, differences in time scalings when using 
the new polarizable water model do emphasize the need to carefully analyze rates determined with these simulations. Furthermore, given the quantitatively similar results obtained using the two MARTINI water models, the use of the polarizable water model to study these phase phenomena likely adds unnecessary computational cost, due to the addition of charged interaction sites in the solvent. We also observe that the combined results of the MARTINI model and the DPD model presented both here and in previous studies $^{11,21}$ suggest that reparametrization of the bead radii for MARTINI lipid heads relative to MARTINI lipid tails may lead to a lipid model that better captures both the thermodynamic signatures and the phase diagrams of saturated lipids. Varying bead diameters would lead to a higher-dimensional parameter space than previously explored in the MARTINI coarse-graining procedure of fitting various solvation free energies via Lennard-Jones $\varepsilon$ while holding the bead diameter $\sigma$ constant, but it holds the promise of better representing the balance between lipid head and tail packing which seems crucial for representing the ripple and tilted gel phases.

Finally, we have found that computational DSC is able to indicate phase transitions in lipid bilayers, in line with its experimental equivalent. The measured transition temperatures and transition enthalpies are relevant quantities to match in further improving coarse-grained lipid models. We do note, however, that either finite-size scaling analysis or umbrella sampling would be merited in matching such quantities for the MARTINI model where the hysteresis between heating and cooling curves is quite substantial. It could also be interesting to evaluate other models with this same methodology.

\section{ASSOCIATED CONTENT}

S Supporting Information. Discussion of the conversion between the reduced units of DPD simulations and the physical units employed in this article. Additional structural results of the DPD model as well as the MARTINI results for simulation with $90 \%$ water beads and $10 \%$ antifreeze beads. This material is available free of charge via the Internet at http://pubs.acs.org.

\section{AUTHOR INFORMATION}

\section{Corresponding Author}

*E-mail: jrodgers78@gmail.com (J.M.R.); berend-smit@berkeley. edu (B.S.).

\section{ACKNOWLEDGMENT}

We thank S.-J. Marrink for his helpful comments. J.M.R. acknowledges the support of the Chemical Sciences, Geosciences and Biosciences Division, Office of Basic Energy Sciences, Office of Science, U.S. Department of Energy, FWP No. SISGRKN. J.S. received support from Innovation Center Denmark, Silicon Valley, the Center for Information Technology in the Interest of Society (CITRIS), and the Hakon Lund Foundation. J.S. and B.S. acknowledge support from The Danish Council for Independent Research (Natural Sciences), the Centre for Theory in Natural Sciences, Aarhus University, and the Danish Center for Scientific Computing. F.M. was supported by the Laboratory Directed Research and Development Program of Lawrence Berkeley National Laboratory under the Department of Energy Contract No. DE-AC02-05CH11231.

\section{REFERENCES}

(1) Coarse-Graining of Condensed Phase and Biomolecular Systems; Voth, G. A., Ed.; CRC Press: Boca Raton, FL, USA, 2009.

(2) Deserno, M. Macromol. Rapid Commun. 2009, 30, 752-771.

(3) Bennun, S. V.; Hoopes, M. I.; Xing, C.; Faller, R. Chem. Phvs. Lipids 2009, 159, 59-66.

(4) Venturoli, M.; Sperotto, M. M.; Kranenburg, M.; Smit, B. Phvs. Rep. 2006, 437, 1-57.

(5) Klauda, J. B.; Venable, R. M.; Freites, J. A.; O'Connor, J. W.; Tobias, D. J.; Mondragon-Ramirez, C.; Vorobyov, I.; MacKerell, J.; Alexander, D.; Pastor, R. W. L.Phvs. Chem. B 2010, 114, 7830-7843.

(6) Tieleman, D.; MacCallum, J.; Ash, W.; Kandt, C.; Xu, Z.; Monticelli, L. I. Phvs.: Condens. Matter 2006, 18, S1221-S1234.

(7) Marrink, S. J.; Risselada, H. J.; Yefimov, S.; Tieleman, D. P.; de Vries, A. H. I. Phvs. Chem. B 2007, 111, 7812-7824.

(8) Shinoda, W.; DeVane, R.; Klein, M. L. L. Phvs. Chem. B 2010, 114, 6836-6849.

(9) Groot, R. D.; Warren, P. B. I. Chem. Phys. 1997, 107, 4423-4435.

(10) Marrink, S. J.; de Vries, A. H.; Mark, A. E. L.Phvs. Chem. B 2004, 108, 750-760.

(11) Kranenburg, M.; Smit, B. L.Phvs. Chem. B 2005, 109, 6553-6563.

(12) Risselada, H. J.; Marrink, S. J. Proc. Natl. Acad. Sci. U.S.A. 2008, 105, 17367-17372.

(13) Schaefer, L. V.; de Jong, D. H.; Holt, A.; Rzepiela, A. J.; de Vries, A. H.; Poolman, B.; Killian, J. A.; Marrink, S. J. Proc. Natl. Acad. Sci.U.S.A. 2011, 108, 1343-1348.

(14) de Meyer, F.; Smit, B. Proc. Natl. Acad. Sci. U.S.A. 2009, 106, 3654-3658.

(15) de Meyer, F. J.-M.; Benjamini, A.; Rodgers, J. M.; Misteli, Y.; Smit, B. L.Phvs. Chem. B 2010, 114, 10451-10461.

(16) de Meyer, F. J.-M.; Venturoli, M.; Smit, B. Biophvs. I. 2008, $95,1851-1865$.

(17) de Meyer, F. J. M.; Rodgers, J. M.; Willems, T. F.; Smit, B. Biophvs. I. 2010, 99, 3629-3638.

(18) Kranenburg, M.; Vlaar, M.; Smit, B. Biophys. I. 2004, 87, 15961605.

(19) Kranenburg, M.; Smit, B. FEBS Lett. 2004, 568, 15-18.

(20) Koynova, R.; Caffrey, M. Biochim. Biophvs. Acta 1998, 1376, 91-145.

(21) Marrink, S. J.; Risselada, J.; Mark, A. E. Chem. Phvs. Lipids 2005, $135,223-244$.

(22) Heimburg, T. Thermal Biophysics of Membranes; 1st ed.; WileyVCH: Weinheim, Germany, 2007.

(23) Lindblom, G.; Rilfors, L. Biochim. Biophys. Acta 1989, 988, 221-256.

(24) Caffrey, M.; Magin, R. L.; Hummel, B.; Zhang, J. Biophys. I. $1990,58,21-29$.

(25) Mabrey, S.; Sturtevant, J. M. Proc. Natl. Acad. Sci. U.S.A. 1976, $11,3862-3866$.

(26) Tada, K.; Goto, M.; Tamai, N.; Matsuki, H.; Kaneshina, S. Chem. Phys. Lipids 2008, 153, 138-143.

(27) Chen, S. C.; Sturtevant, J. M.; Gaffney, B. J. Proc. Natl. Acad. Sci. U.S.A. 1980, 77, 5060-5063.

(28) Tristram-Nagle, S.; Nagle, J. F. Chem. Phvs. Lipids 2004, 127, 3-14.

(29) Nagle, J. F.; Wilkinson, D. A. Biophys. I. 1978, 23, 159-175.

(30) Evans, E.; Kwok, R. Biochemistry 1982, 21, 4874-4879.

(31) Heimburg, T.; Würz, U.; Marsh, D. Biophvs. I. 1992, 63, 13691378.

(32) Nagle, J. F.; Tristram-Nagle, S. Biochim. Biophys. Acta 2000, 1469, 159-195.

(33) O'Neill, M. I. Anal. Chem. 1966, 38, 1331-1336.

(34) Ladbrooke, B. D.; Williams, R. M.; Chapman, D. Biochim. Biophvs. Acta 1968, 150, 333-340.

(35) Sugar, I. P.; Biltonen, R. L.; Mitchard, N. Methods Enzvmol. 1994, 240, 569-593.

(36) Almeida, P. F. F. Biochim. Biophys. Acta 2009, 1788, 72-85. 
(37) Zhang, Z.; Zuckermann, M. J.; Mouristen, O. G. Phvs. Rev. A 1993, 46, 6707-6713.

(38) Heimburg, T. Biophys. I. 2000, 78, 1154-1165.

(39) Hömberg, M.; Müller, M. J. Chem. Phys. 2010, 132, 155104 $1-17$.

(40) Venturoli, M.; Smit, B. PhvsChemComm 1999, 2, 45-49.

(41) Groot, R. D. Langmuir 2000, 16, 7493-7502.

(42) Kranenburg, M.; Venturoli, M.; Smit, B. Phvs. Rev. E 2003, 67, 060901(R).

(43) Kranenburg, M.; Laforge, C.; Smit, B. Phvs. Chem. Chem. Phvs. 2004, 6, 4531-4534.

(44) Yesylevskyy, S. O.; Schäfer, L. V.; Sengupta, D.; Marrink, S. I. PLoS Comput. Biol. 2010, 6, e1000810.

(45) Feller, S. E.; Pastor, R. W.; Rojnuckarin, A.; Bogusz, S.; Brooks, B. R. I. Chem. Phvs. 1996, 100, 17011-17020.

(46) Rodgers, J. M.; Weeks, J. D. Proc. Natl. Acad. Sci. U.S.A. 2008, 105, 19136-19141.

(47) Frenkel, D.; Smit, B. Understanding Molecular Simulations: from Algorithms to Applications; 2nd ed.; Academic Press: San Diego, CA, USA, 2002.

(48) Hess, B.; Kutzner, C.; van der Spool, D.; Lindahl, E. L. Chem. Theorv Comput. 2008, 4, 435-447.

(49) Español, P.; Warren, P. B. Europhys. Lett. 1995, 30, 191-196.

(50) Rodgers, J. M.; Webb, M.; Smit, B. I. Chem. Phys. 2010, 132, 064107.

(51) Allen, M. P. I. Phvs. Chem. B 2006, 110, 3823-3830.

(52) Berendsen, H. J. C.; Postma, J. P. M; van Gunsteren, W. F.; Di Nola, A.; Haak, J. R. I. Chem. Phys. 1984, 81, 3684-3690.

(53) Rodgers, J. M.; Smit, B. J. Chem. Theorv Comput. 2012, accepted for publication, DOI: $10.1021 /$ ct2007204.

(54) Marrink, S.-J. MARTINI: Biomolecular Forcefield for Coarse Grained Simulations. http://cgmartini.nl (accessed March 2011).

(55) Winger, M.; Trzesniak, D.; Baron, R.; van Gunsteren, W. F. Phvs. Chem. Chem. Phvs. 2009, 11, 1934-1941.

(56) Marrink, S. J.; Periole, X.; Tieleman, D. P.; de Vries, A. H. Phvs. Chem. Chem. Phys. 2010, 12, 2254-2256.

(57) Groot, R. D.; Rabone, K. L. Biophys. I. 2001, 81, 725-736.

(58) Almeida, P. F. F.; Vaz, W. L. C.; Thompson, T. E. Biochemistry 1992, 31, 6739-6747.

(59) Gao, L.; Shillcock, J.; Lipowsky, R. I. Chem. Phvs. 2007, 126, 015101.

(60) Müller, A.; Lonsdale, K. Acta Crystallogr. 1948, 1, 129-131.

(61) When considering the electrostatic calculations in general as well as the additional sites on the polarizable MARTINI water model, the speedup for DPD would be greater, but simultaneously the neighbor list implementation in GROMACS should be faster for pair interaction evaluation than the cell list implementation in our in-house DPDMC code. 